\title{
Supporting Information - Bidentate Ligands Capable of Variable Bond Angles in the Self-Assembly of Discrete Supramolecules
}

Ki-Whan Chi, ${ }^{\dagger \dagger}$ Chris Addicott, ${ }^{*}$ Atta M. Arif, ${ }^{\ddagger}$ Neeladri Das, ${ }^{\ddagger}$ and Peter J. Stang ${ }^{*{ }^{\ddagger}}$

${ }^{\ddagger}$ Department of Chemistry, University of Utah, 315 S. 1400 E. Salt Lake City, Utah 84112

${ }^{\dagger}$ Visiting Professor from the Department of Chemistry, University of Ulsan, Ulsan 680-

749 Republic of Korea.

stang@chem.utah.edu

\section{Table of Contents}

Figure S1. ${ }^{31} \mathrm{P}$ NMR of $\mathbf{2 a}$.

Figure S2. ${ }^{1} \mathrm{H}$ NMR of $\mathbf{2 a}$.

Figure S3. ${ }^{31} \mathrm{P}$ NMR of $\mathbf{2 b}$.

Figure S4. ${ }^{1} \mathrm{H}$ NMR of $\mathbf{2 b}$.

Figure S5. ESI-MS of $\mathbf{2 b}$.

Figure S6. ${ }^{31} \mathrm{P}$ NMR of $\mathbf{3 a}$.

Figure S7. ${ }^{1} \mathrm{H}$ NMR of $3 \mathbf{a}$.

Figure S8. ESI-MS of $\mathbf{3 a}$.

Figure S9. ${ }^{31} \mathrm{P}$ NMR of $\mathbf{3 b}$.

Figure S10. ${ }^{1} \mathrm{H}$ NMR of $\mathbf{3 b}$.

Figure S11. ESI-MS of $\mathbf{3 b}$.

Figure S12. ${ }^{31} \mathrm{P}$ NMR of $\mathbf{4 a}$.

Figure S13. ${ }^{1} \mathrm{H}$ NMR of $\mathbf{4 a}$.

Figure S14. ESI-MS of $\mathbf{4 a}$.

Figure S15. ${ }^{31} \mathrm{P}$ NMR of $\mathbf{4 b}$.

Figure S16. ${ }^{1} \mathrm{H}$ NMR of $\mathbf{4 b}$.

Figure S17. ESI-MS of $\mathbf{4 b}$.

Figure S18. ${ }^{31} \mathrm{P}$ NMR spectra of $\mathbf{2 b}$ at $20{ }^{\circ} \mathrm{C}$ (top). and $80{ }^{\circ} \mathrm{C}$.

Figure S19. ${ }^{1} \mathrm{H}$ NMR spectra of $\mathbf{2 b}$ at $20{ }^{\circ} \mathrm{C}$ (top). and $80{ }^{\circ} \mathrm{C}$.

Table S1 - S6. Crystal data and structure refinement for $\mathbf{2 b}$. 

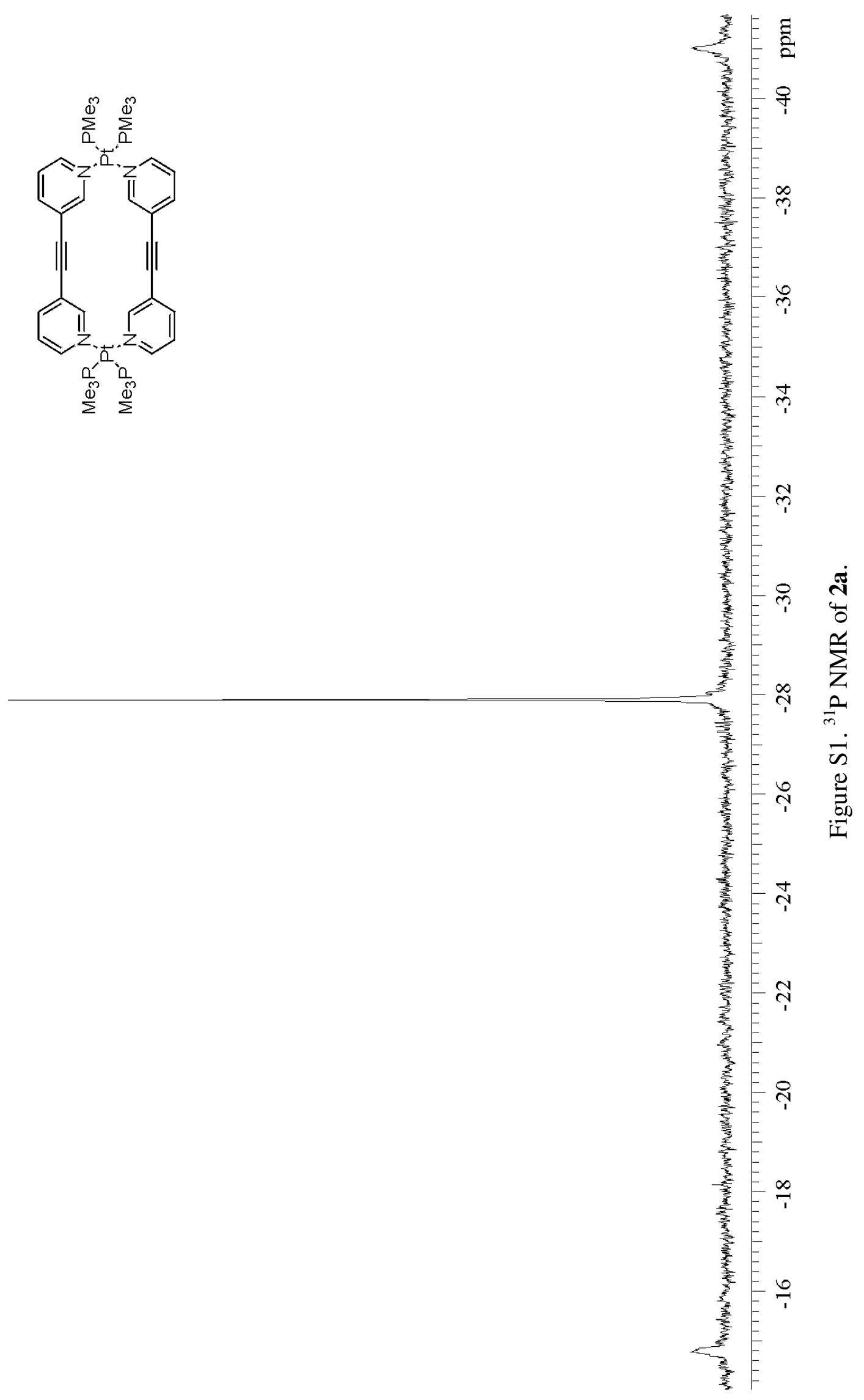

ชี 


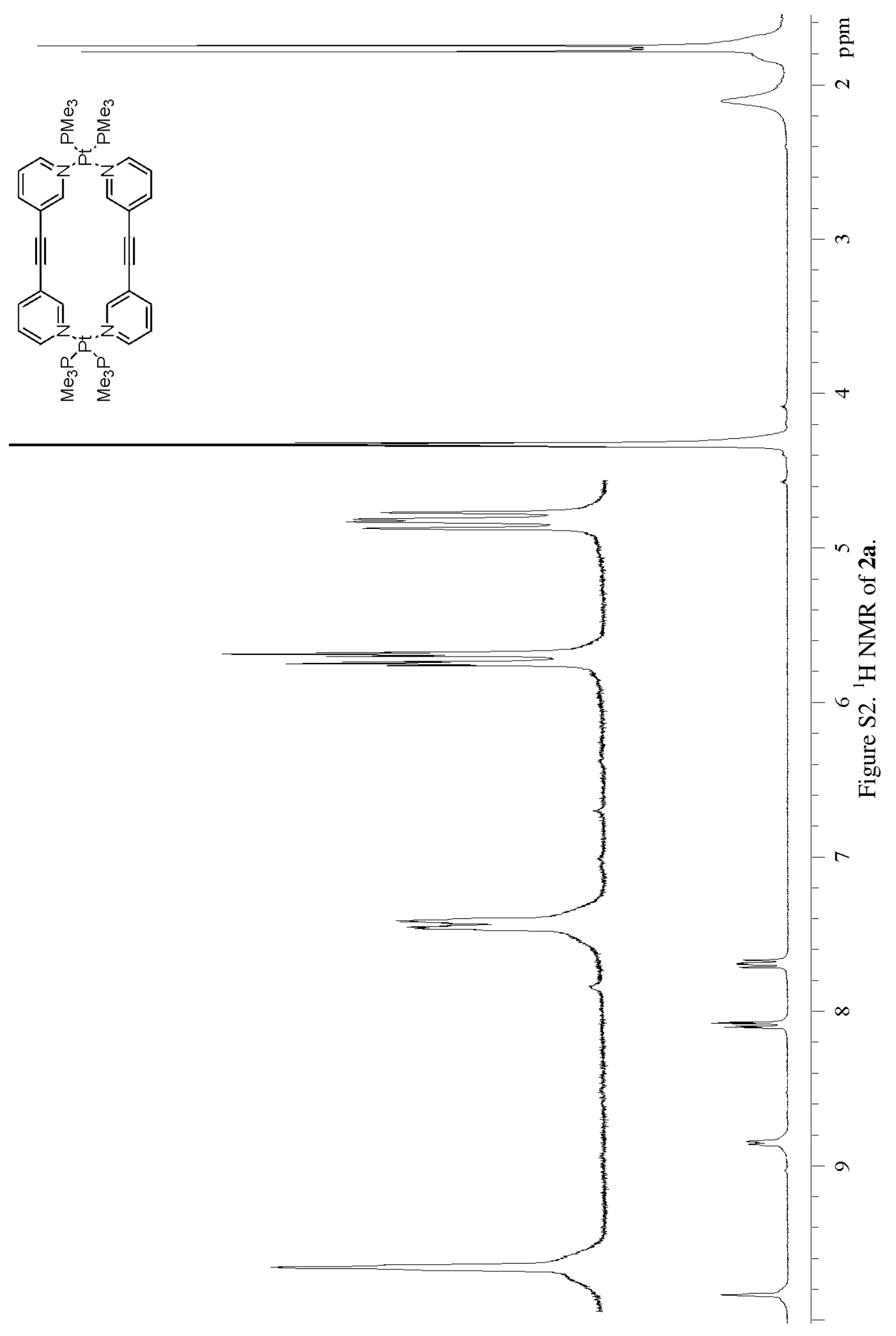




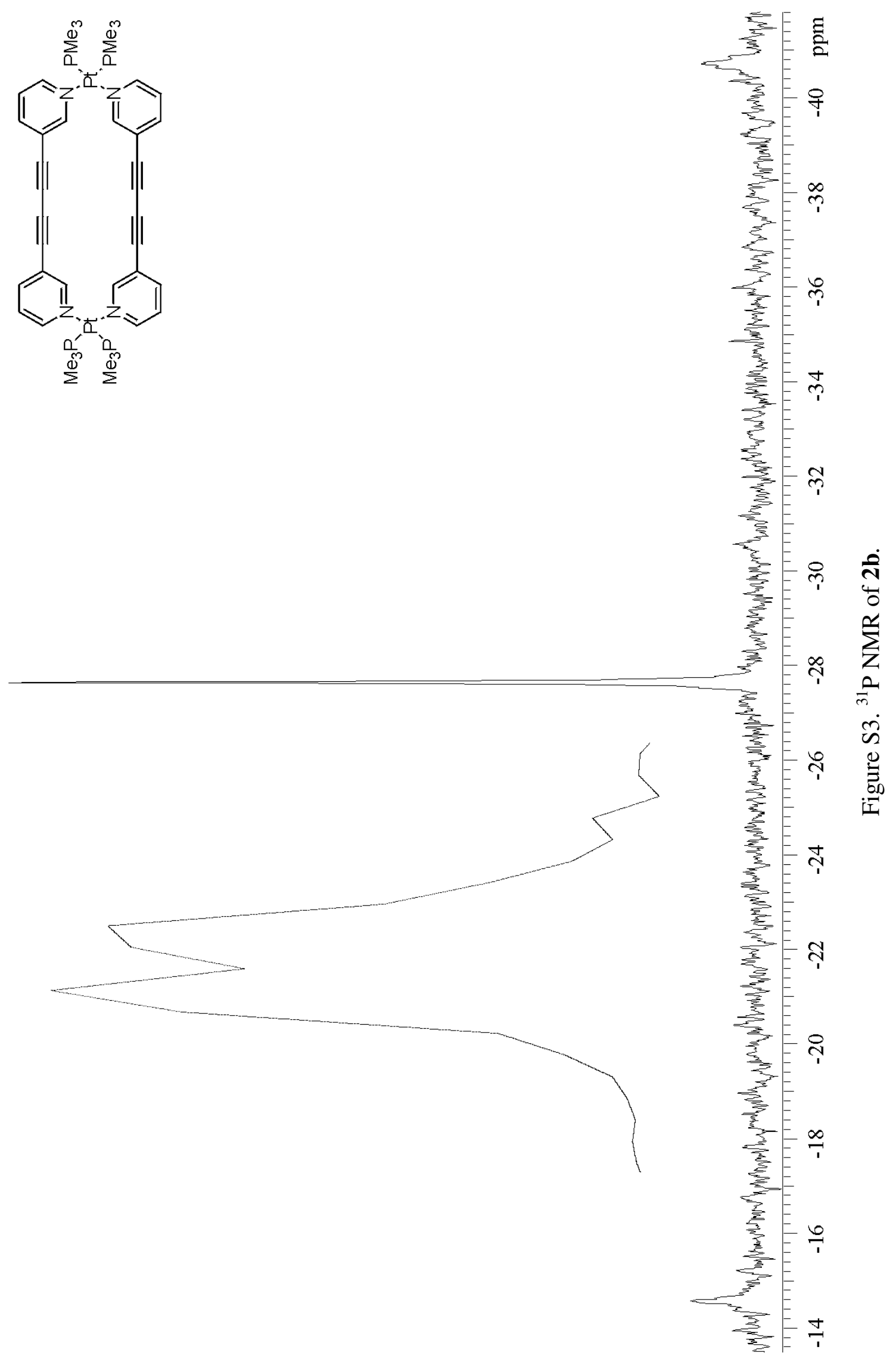




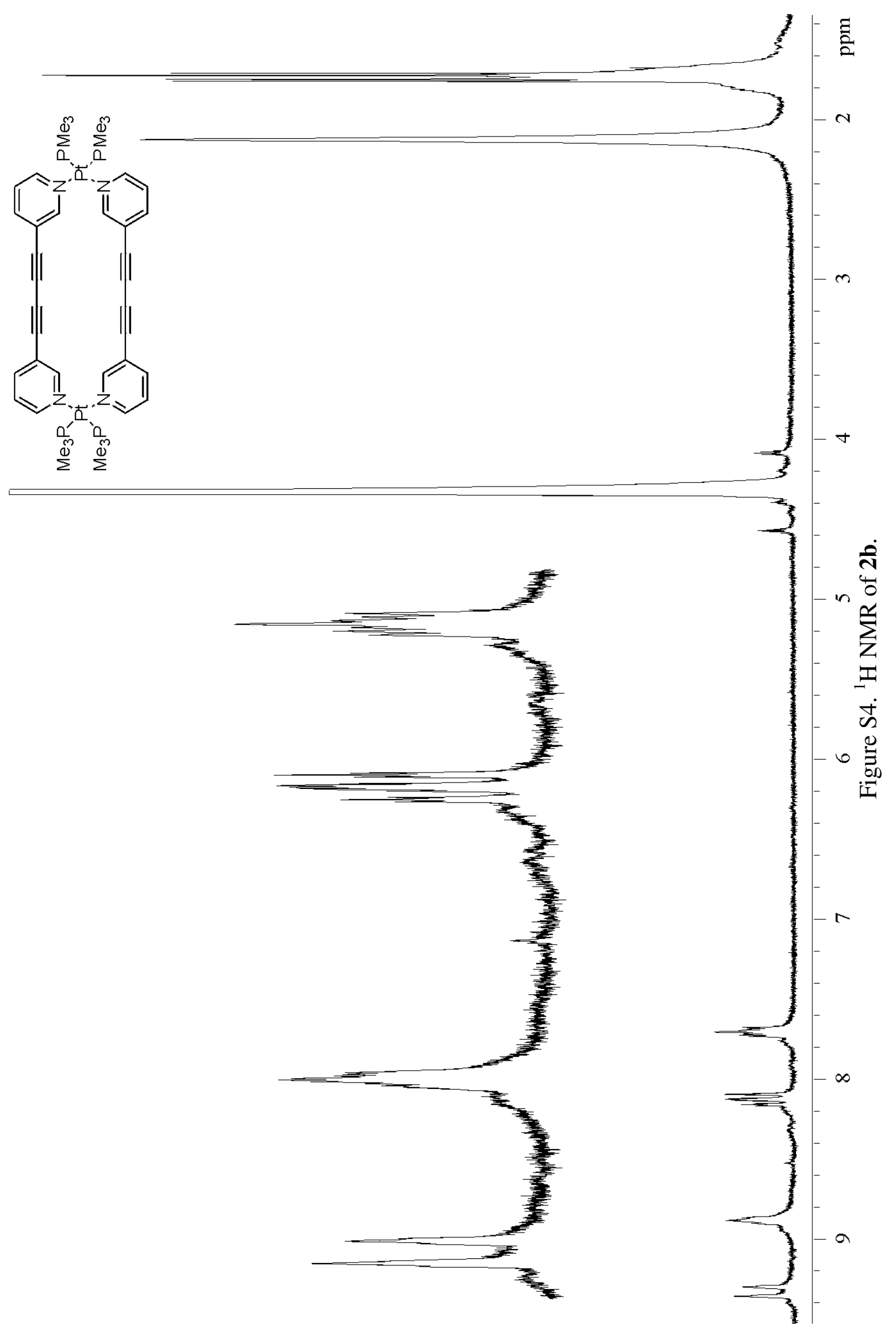




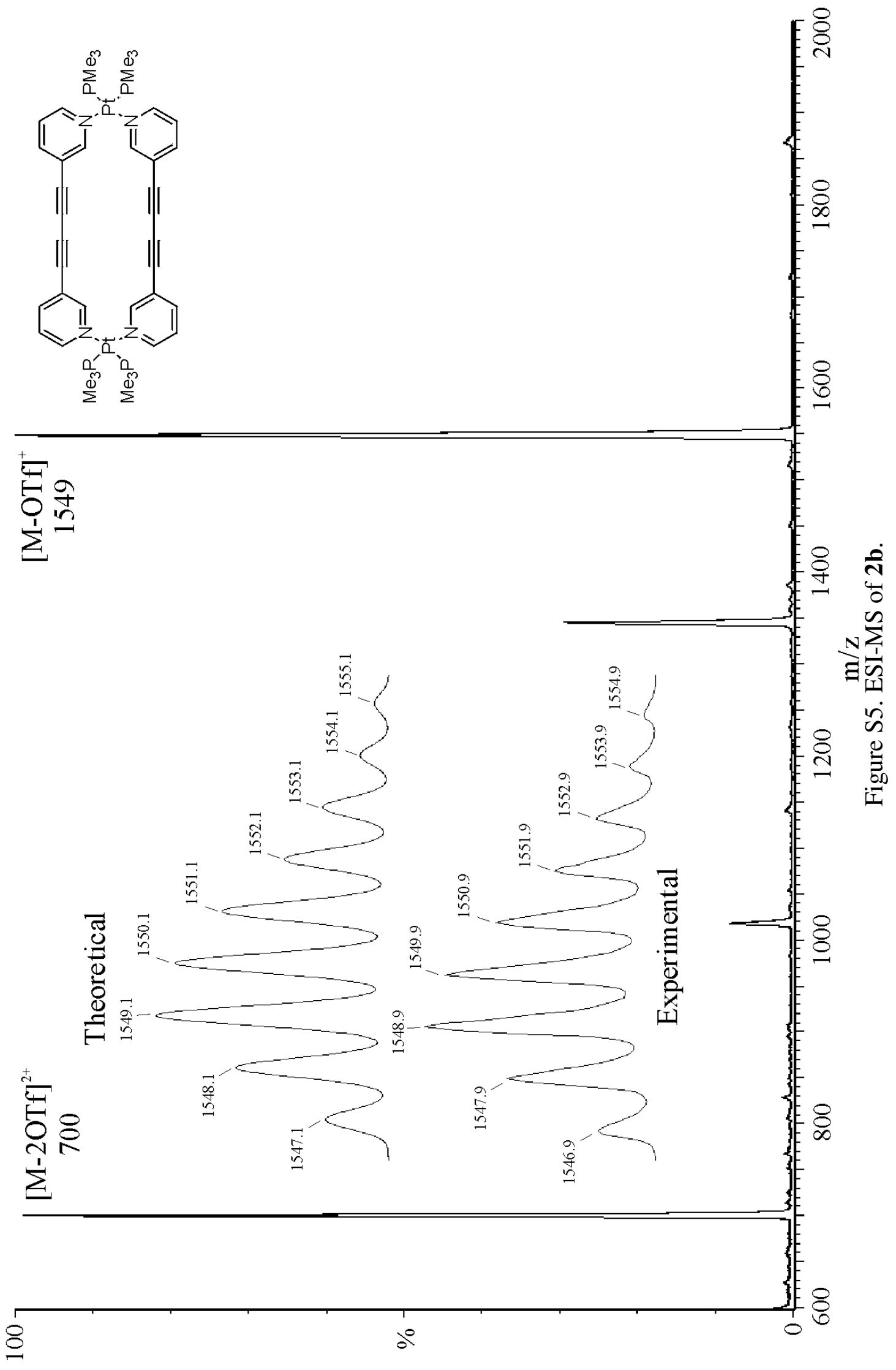



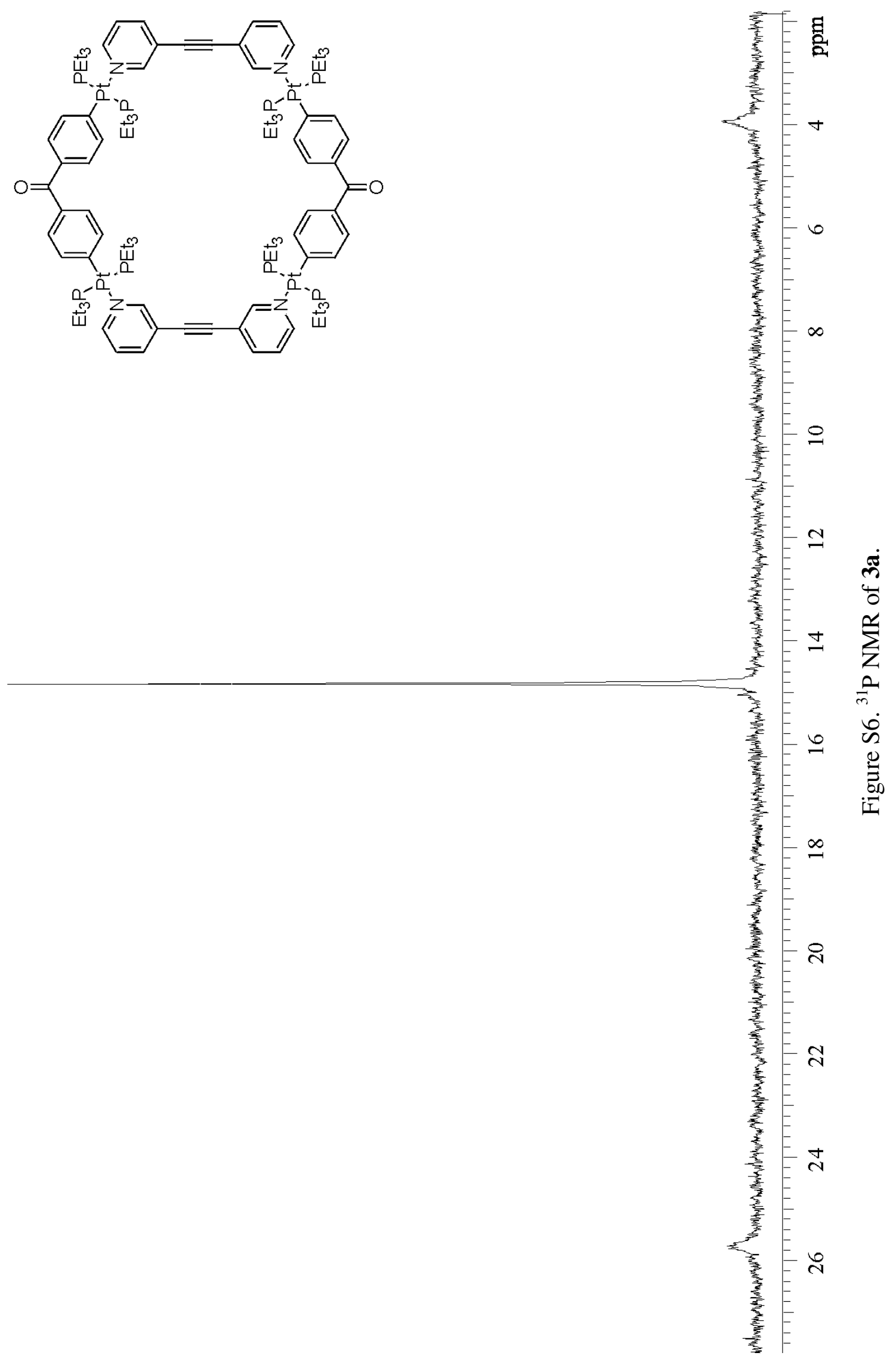

$\tilde{\sigma}$ 


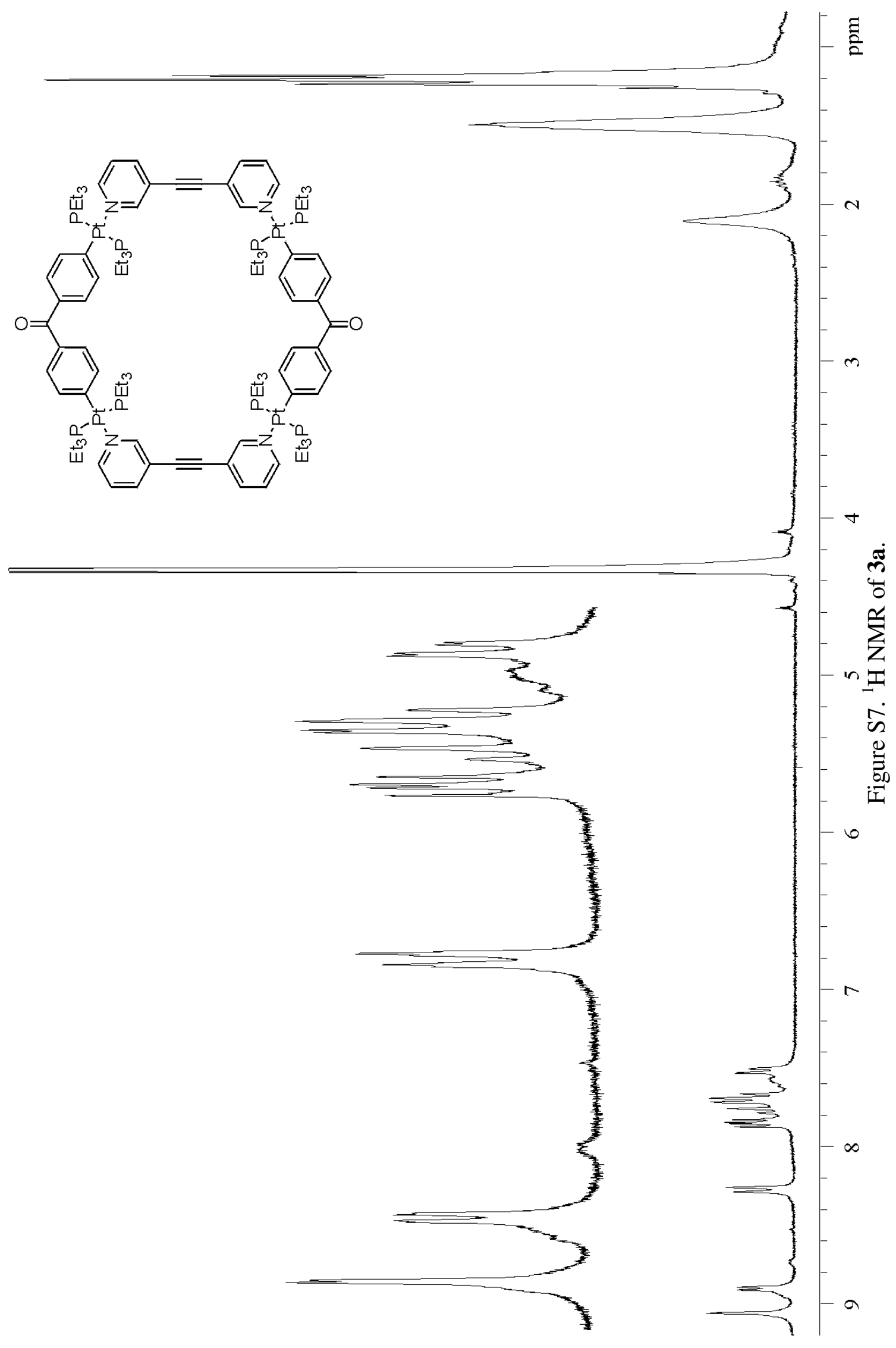




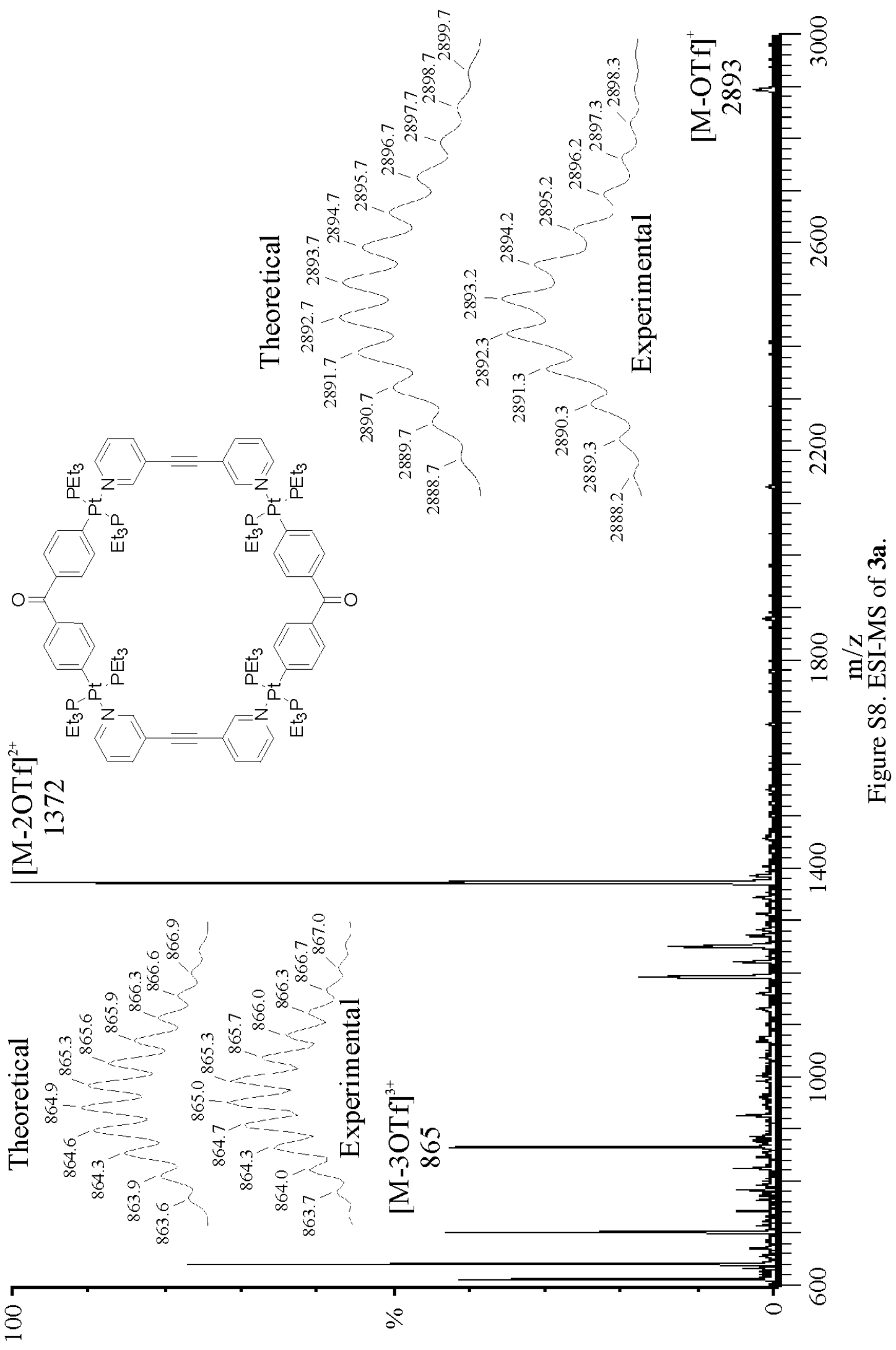



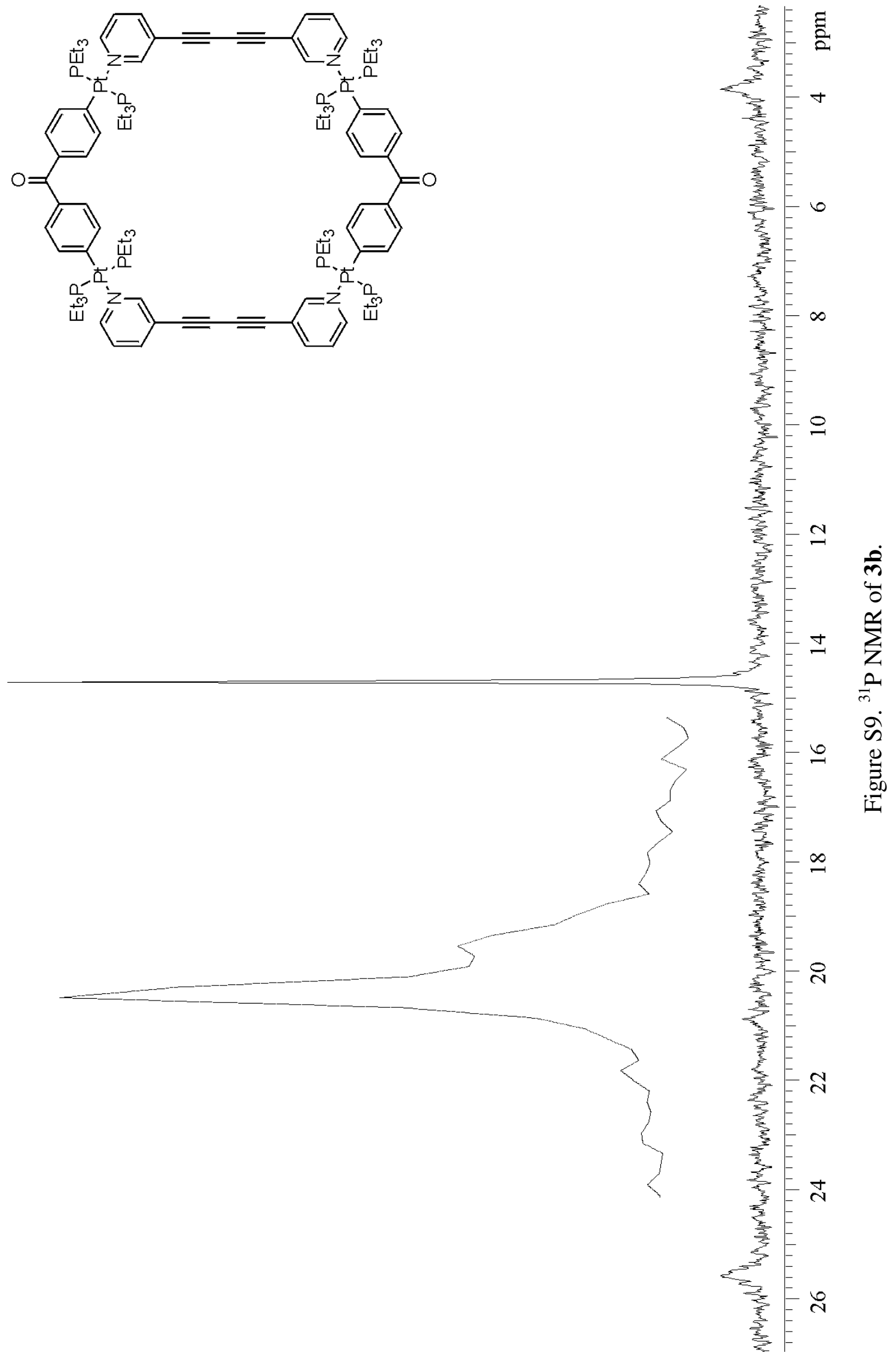


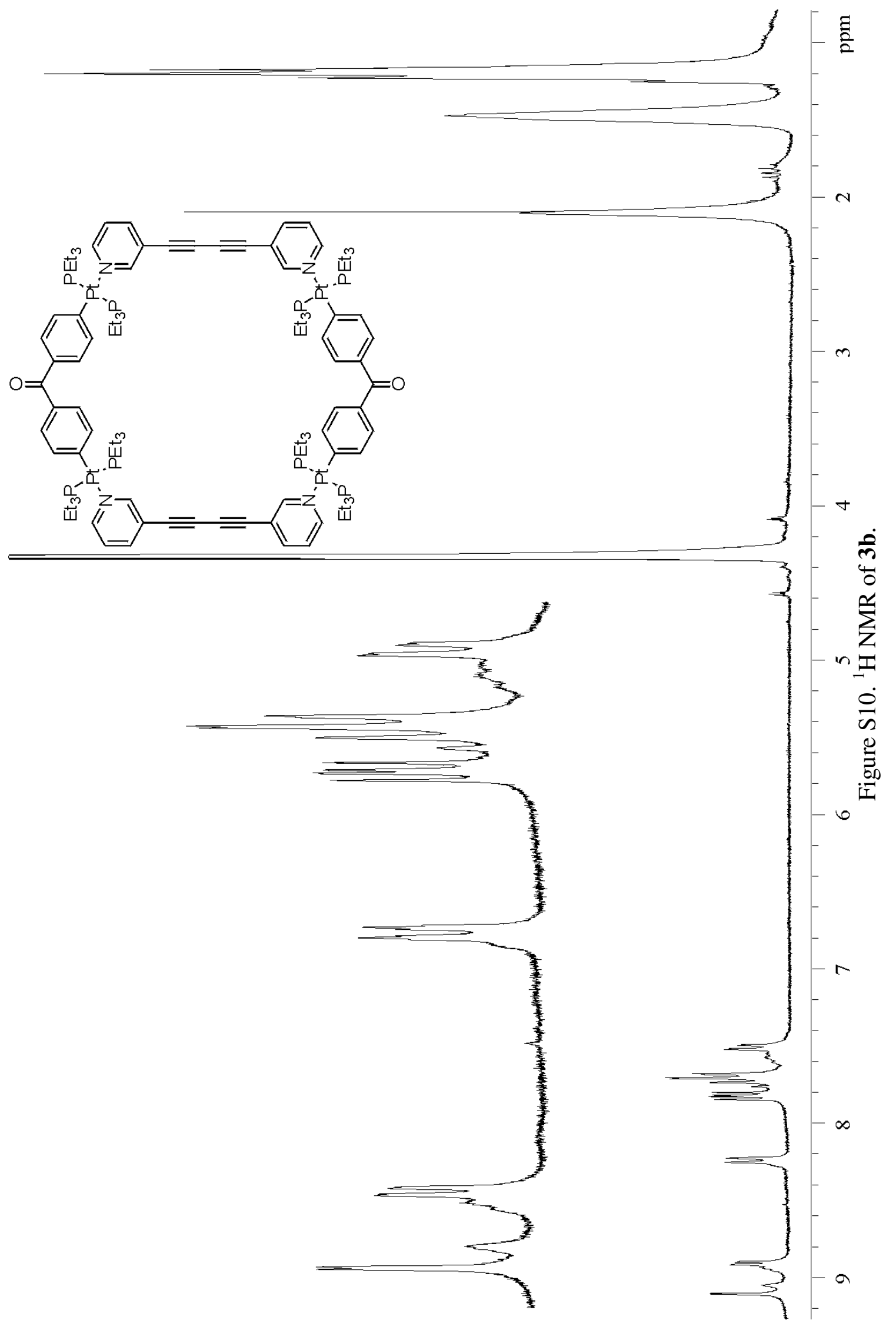



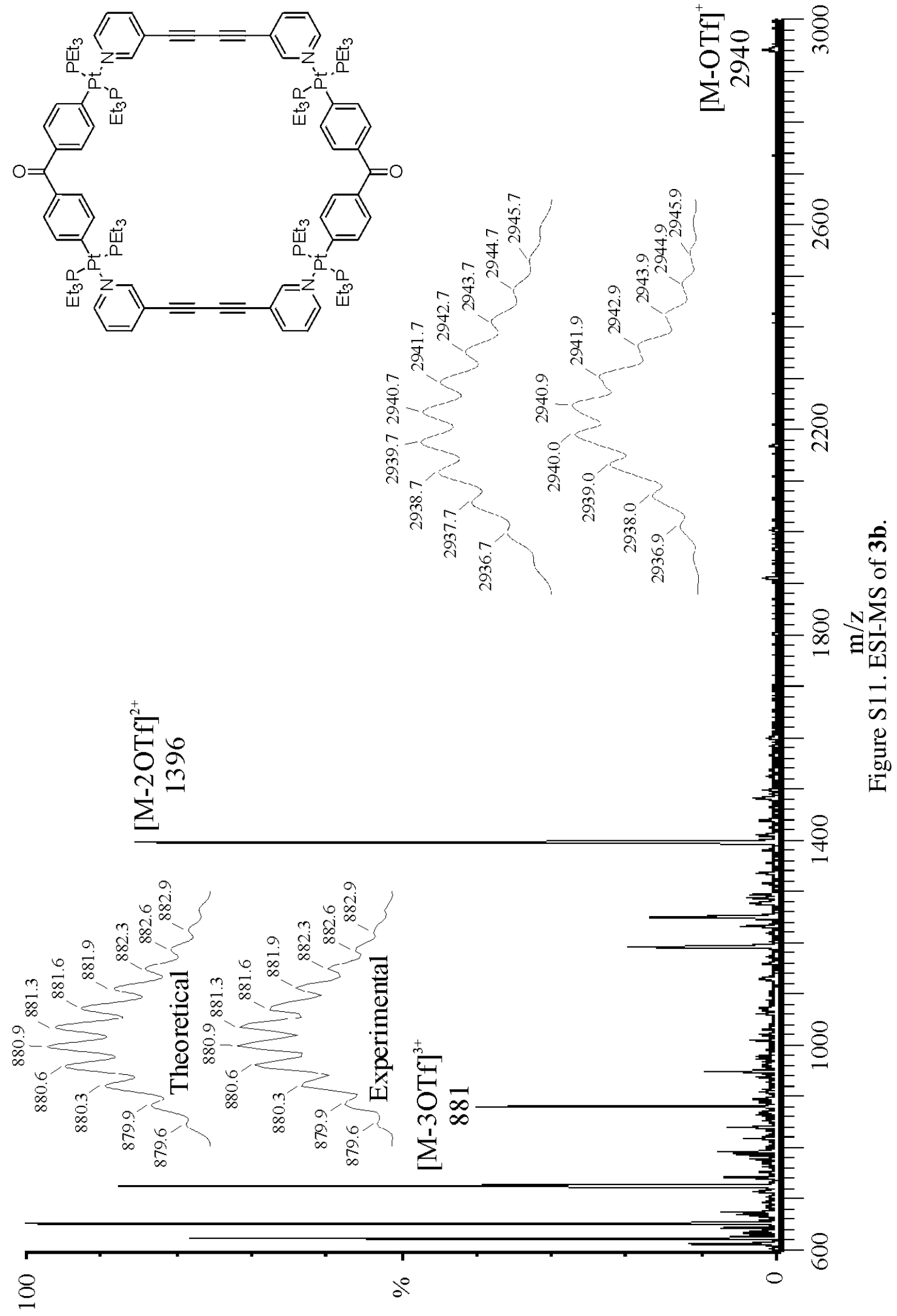


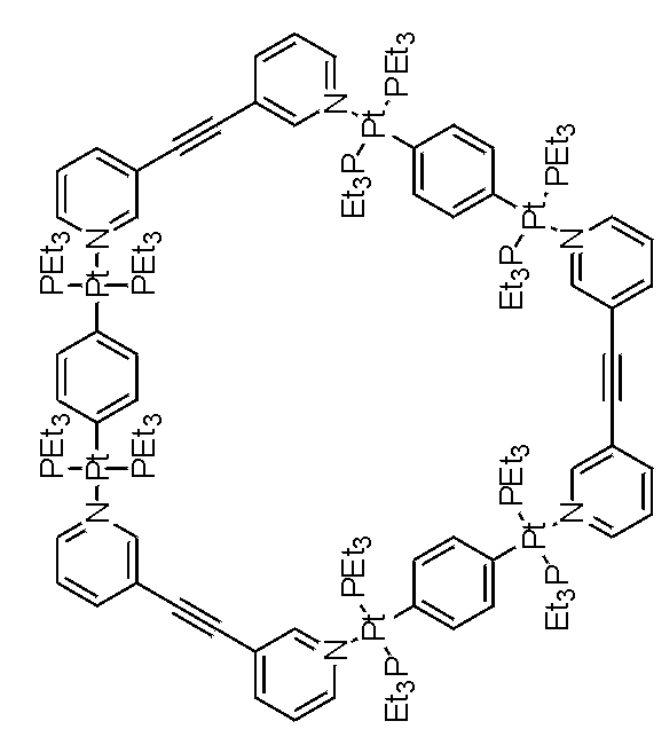

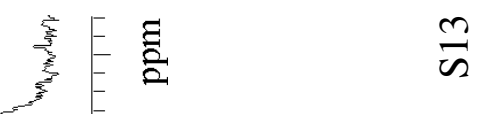

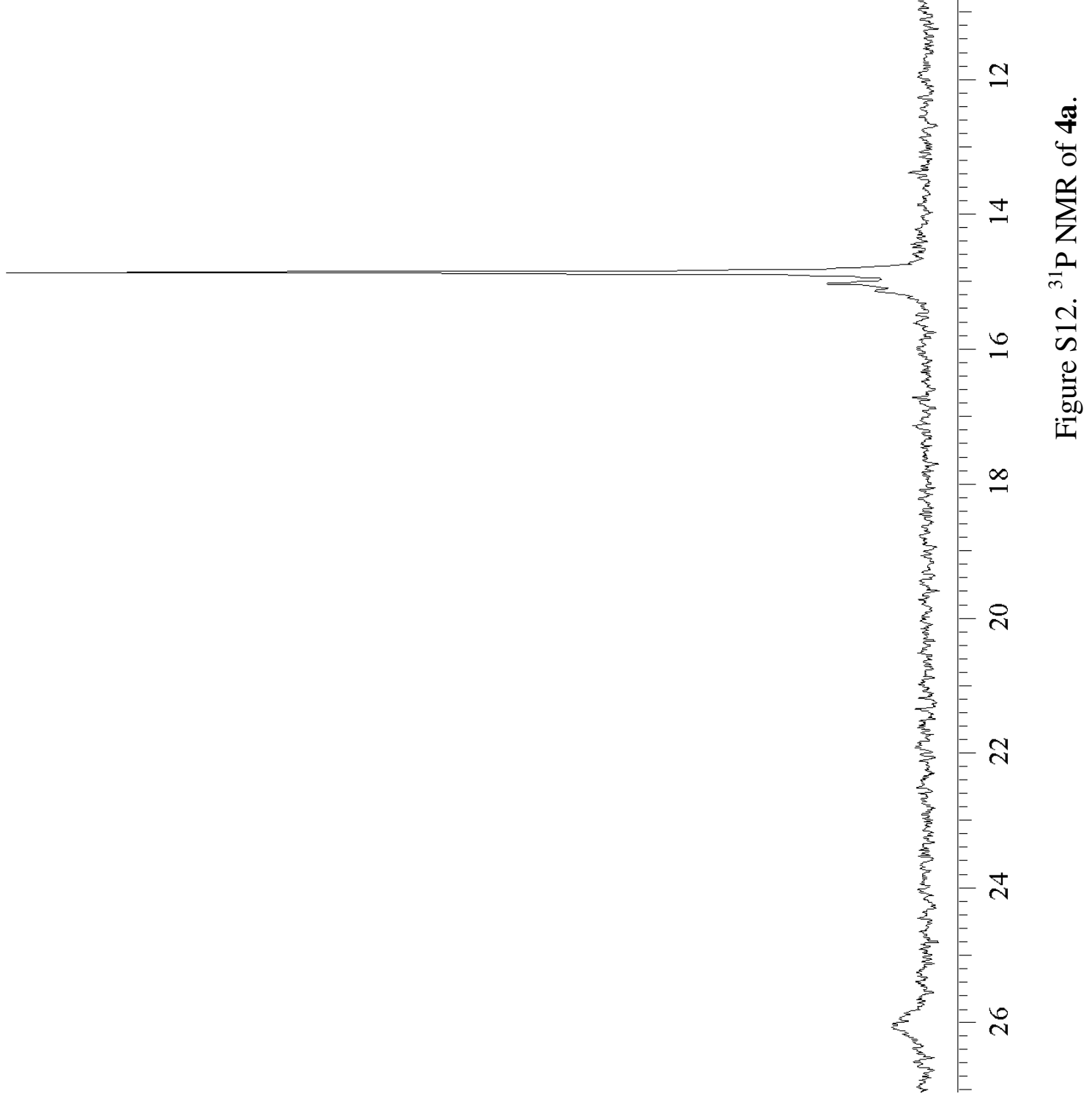




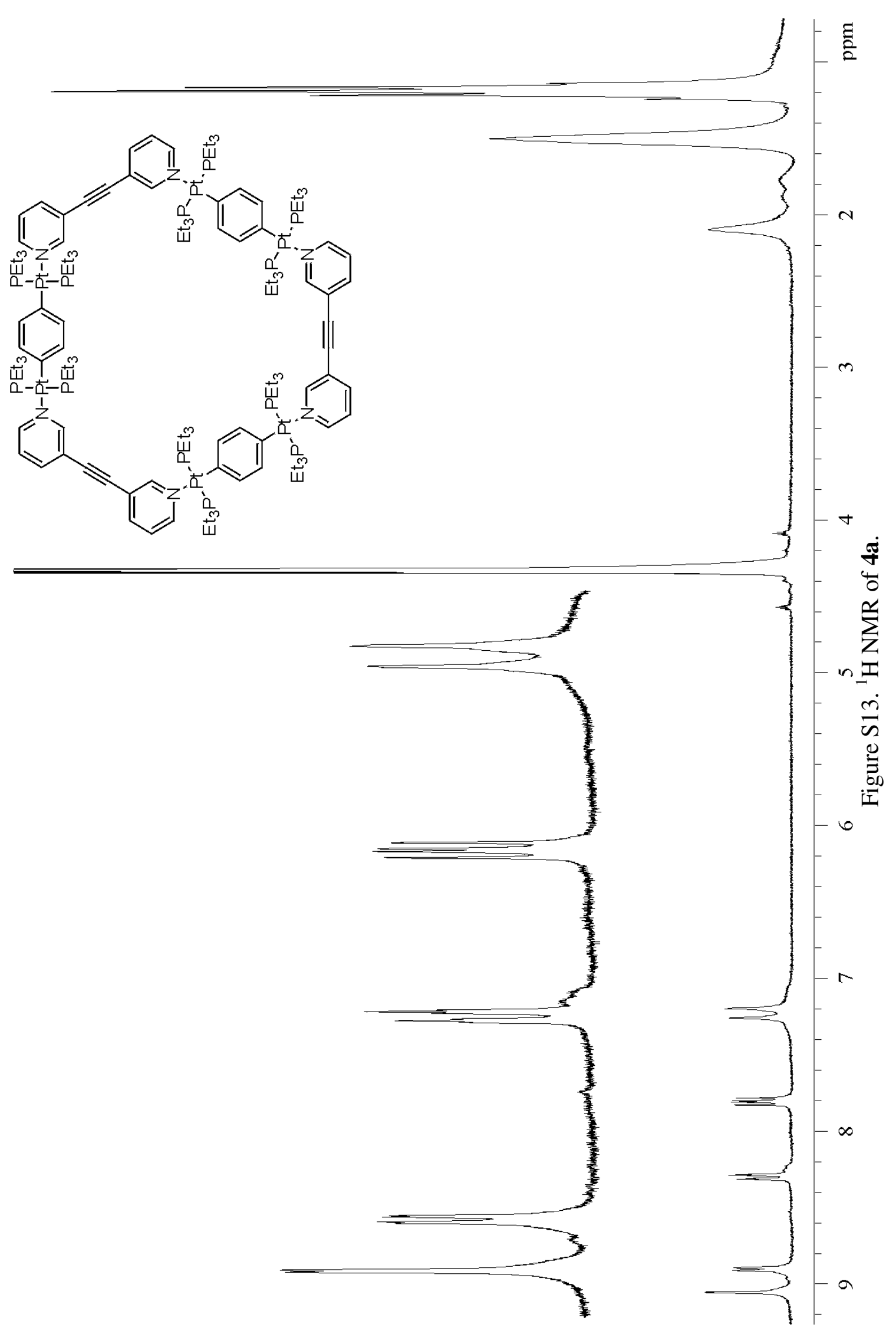




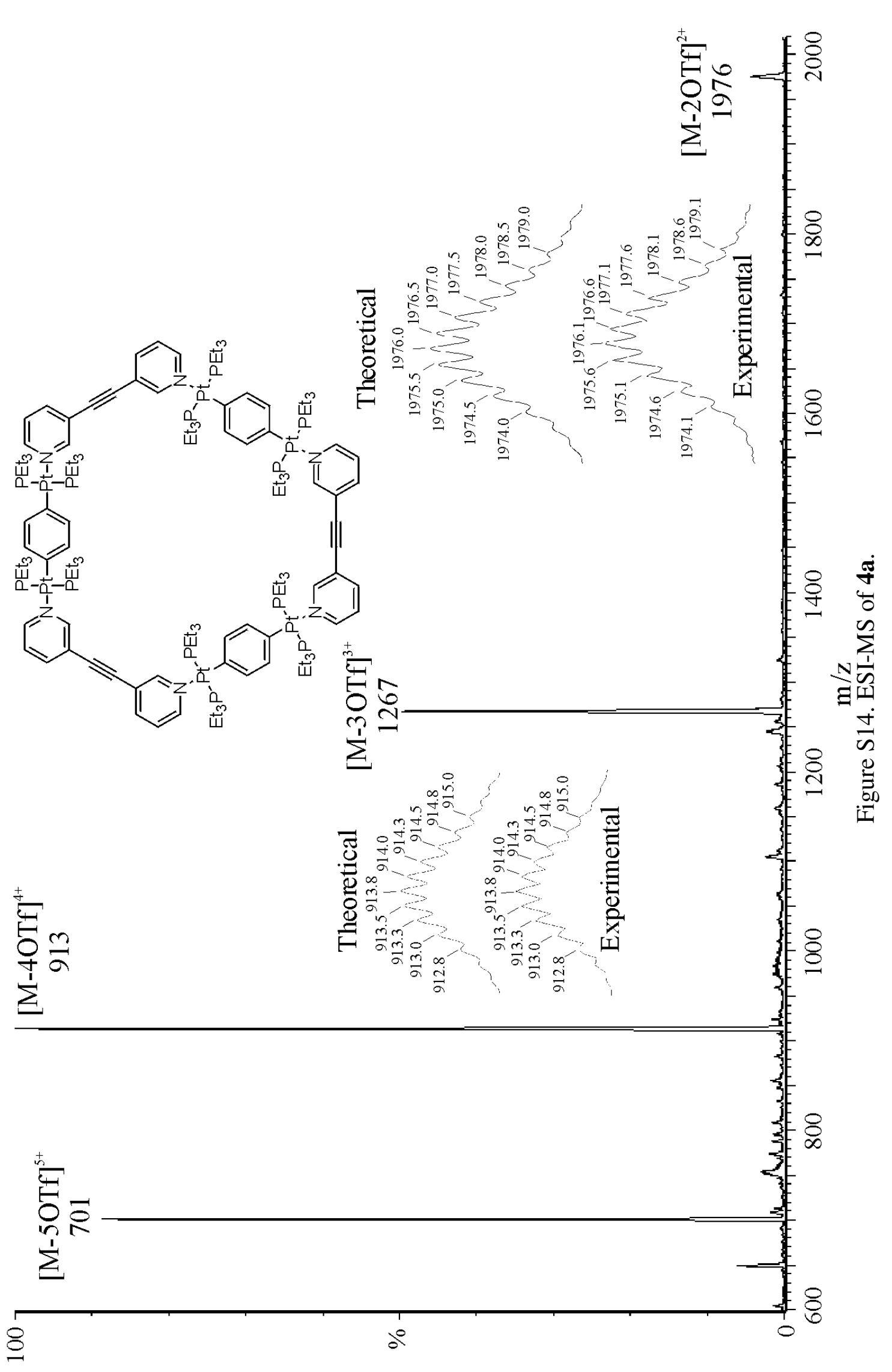




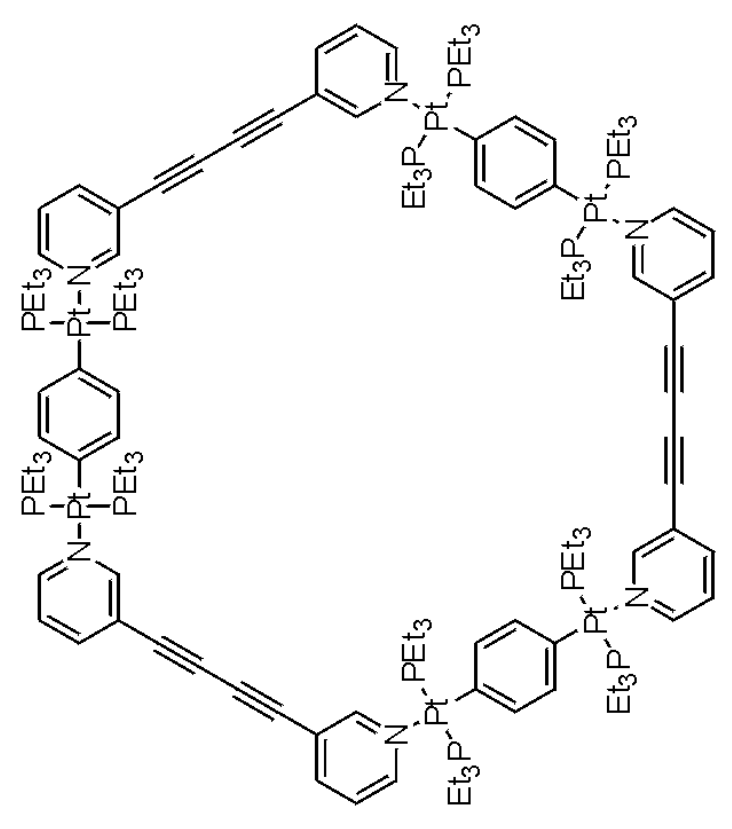

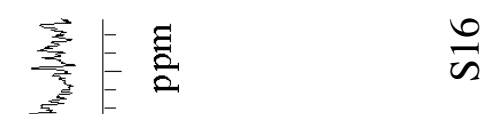

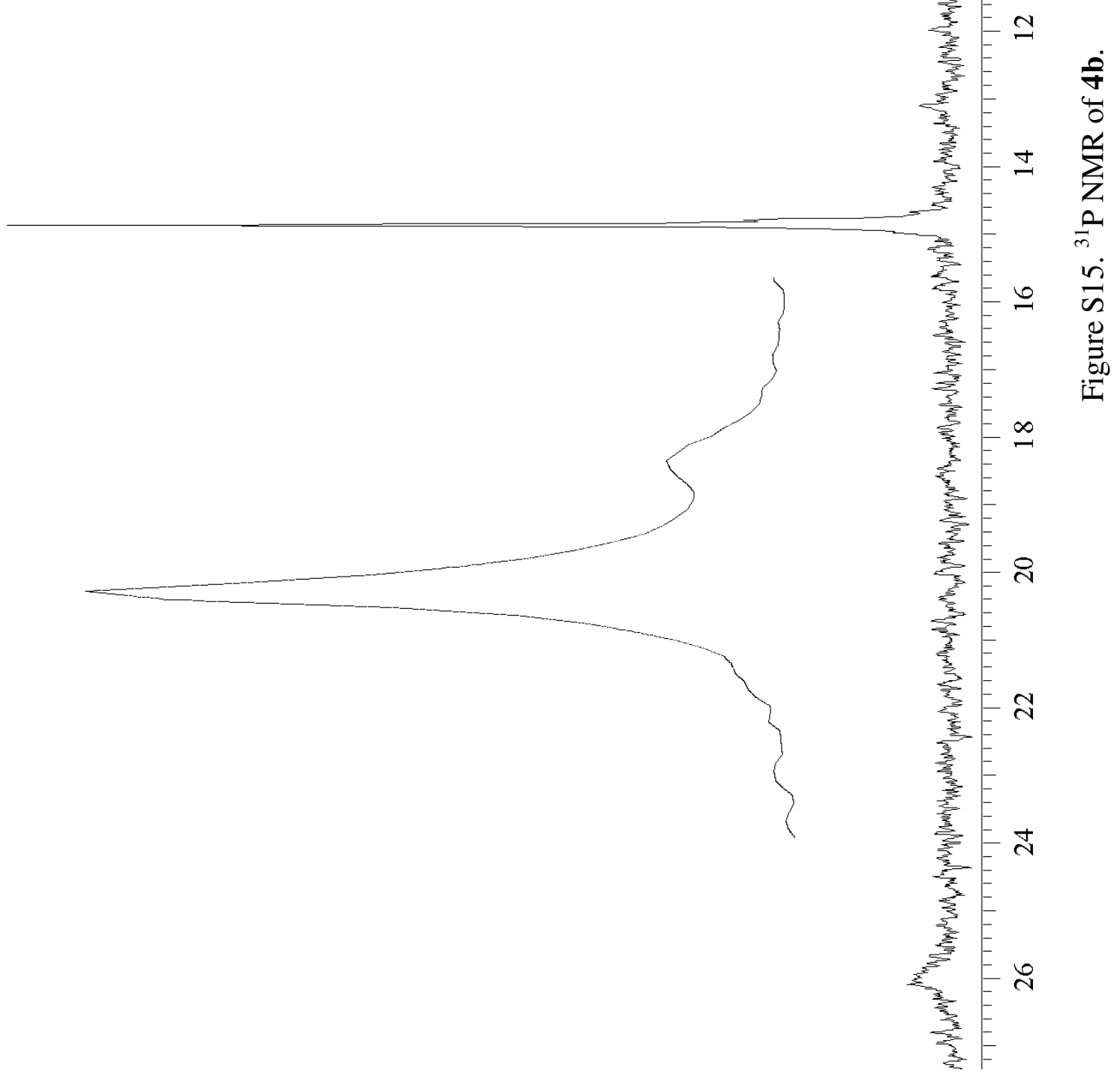




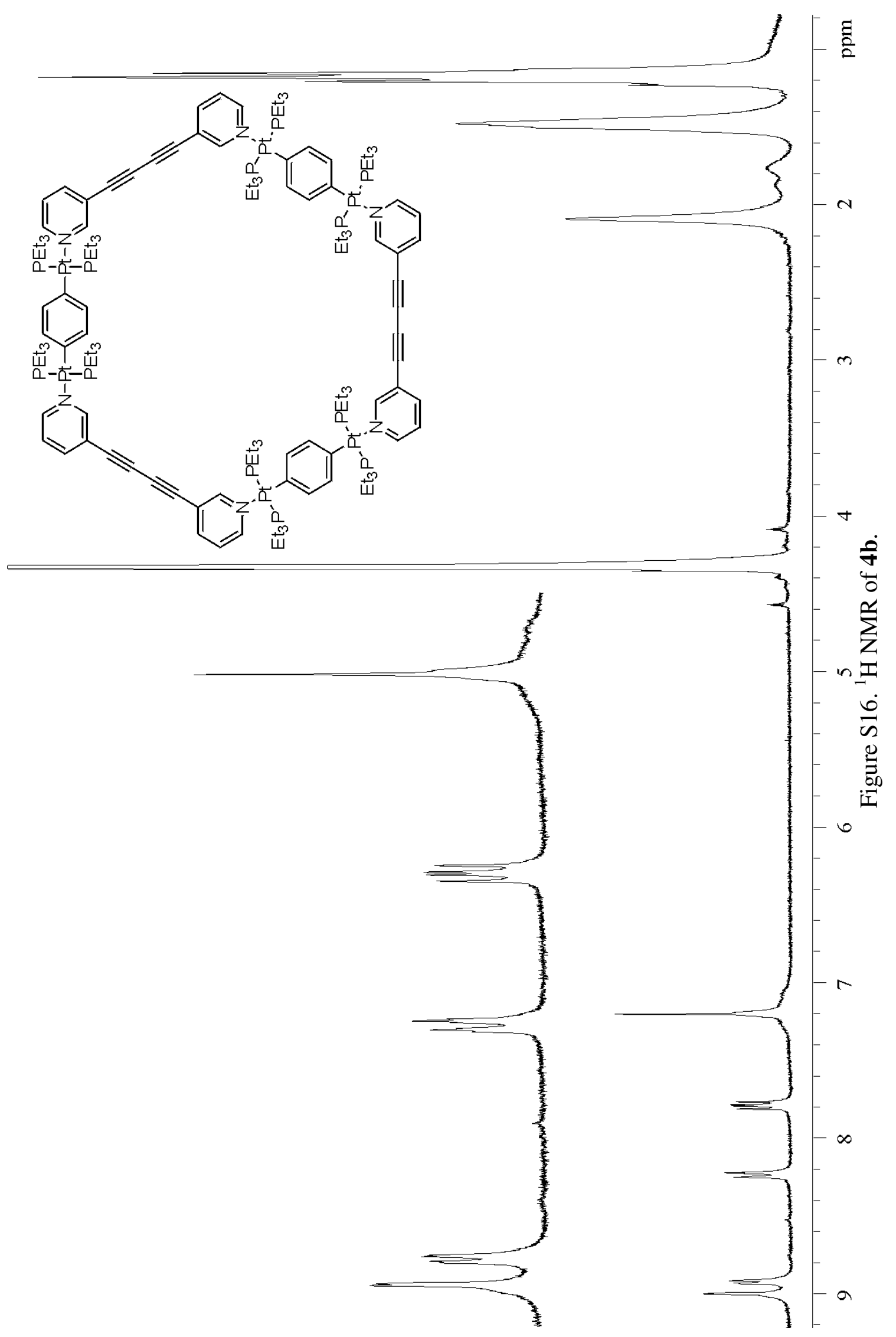




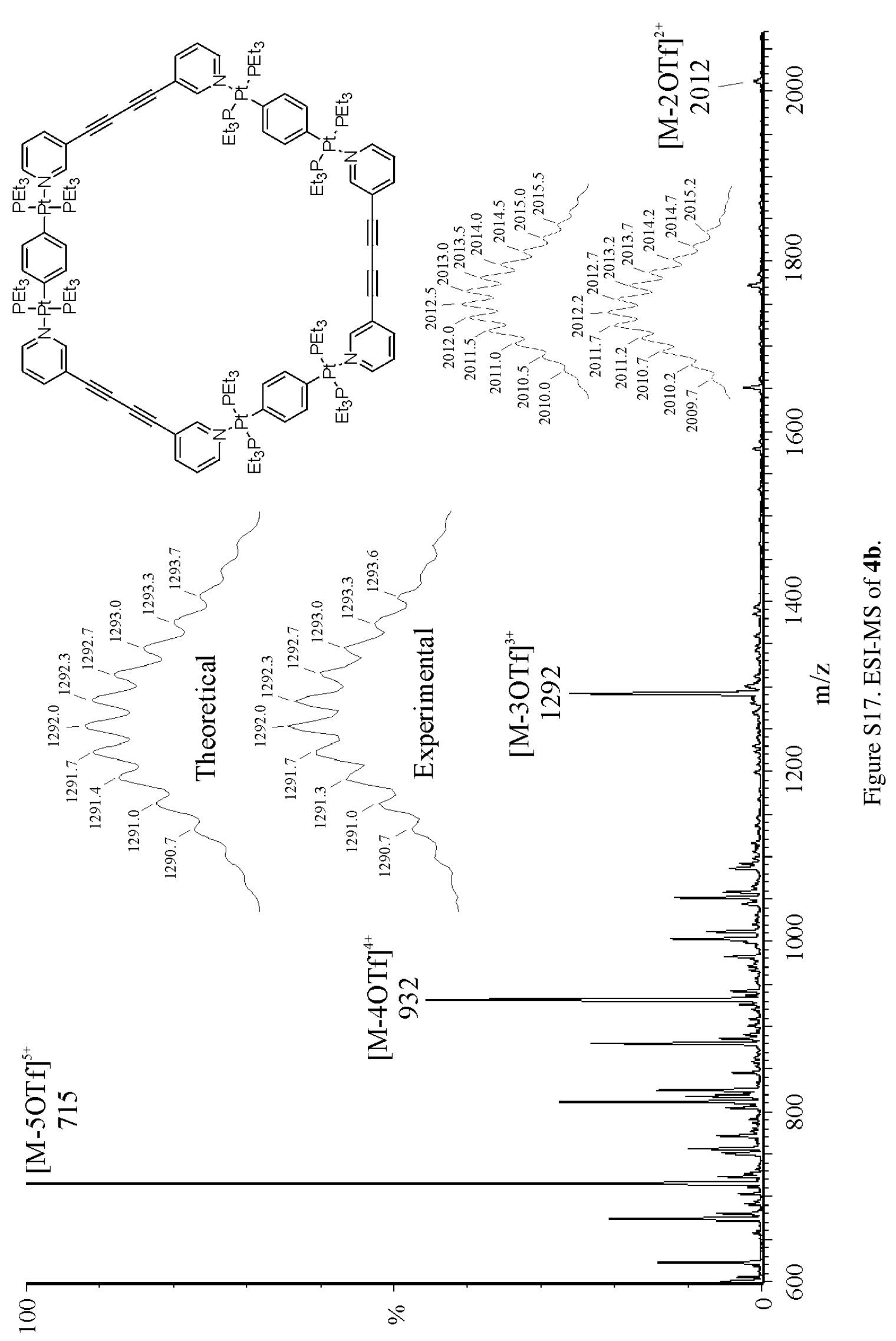




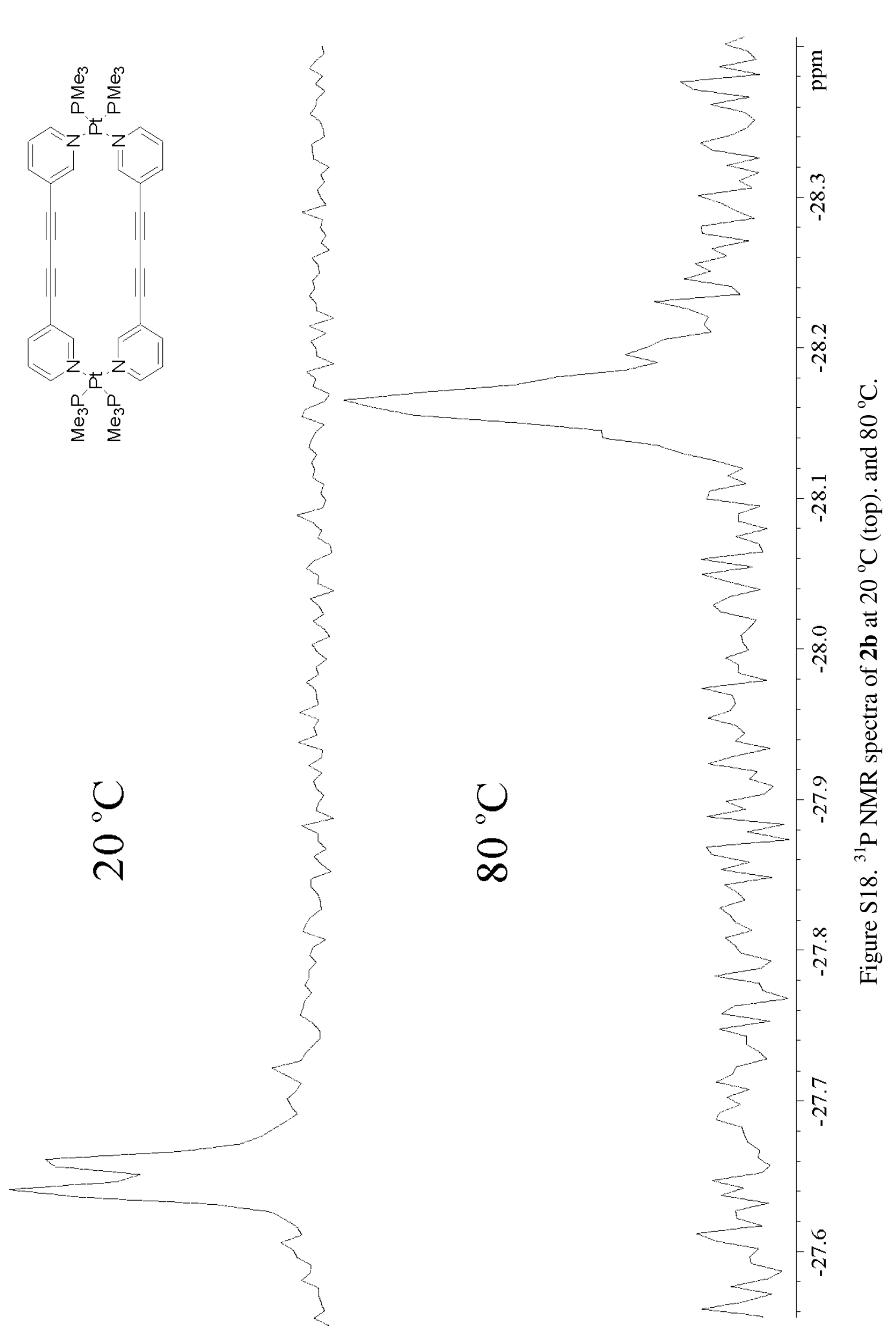


ฌั

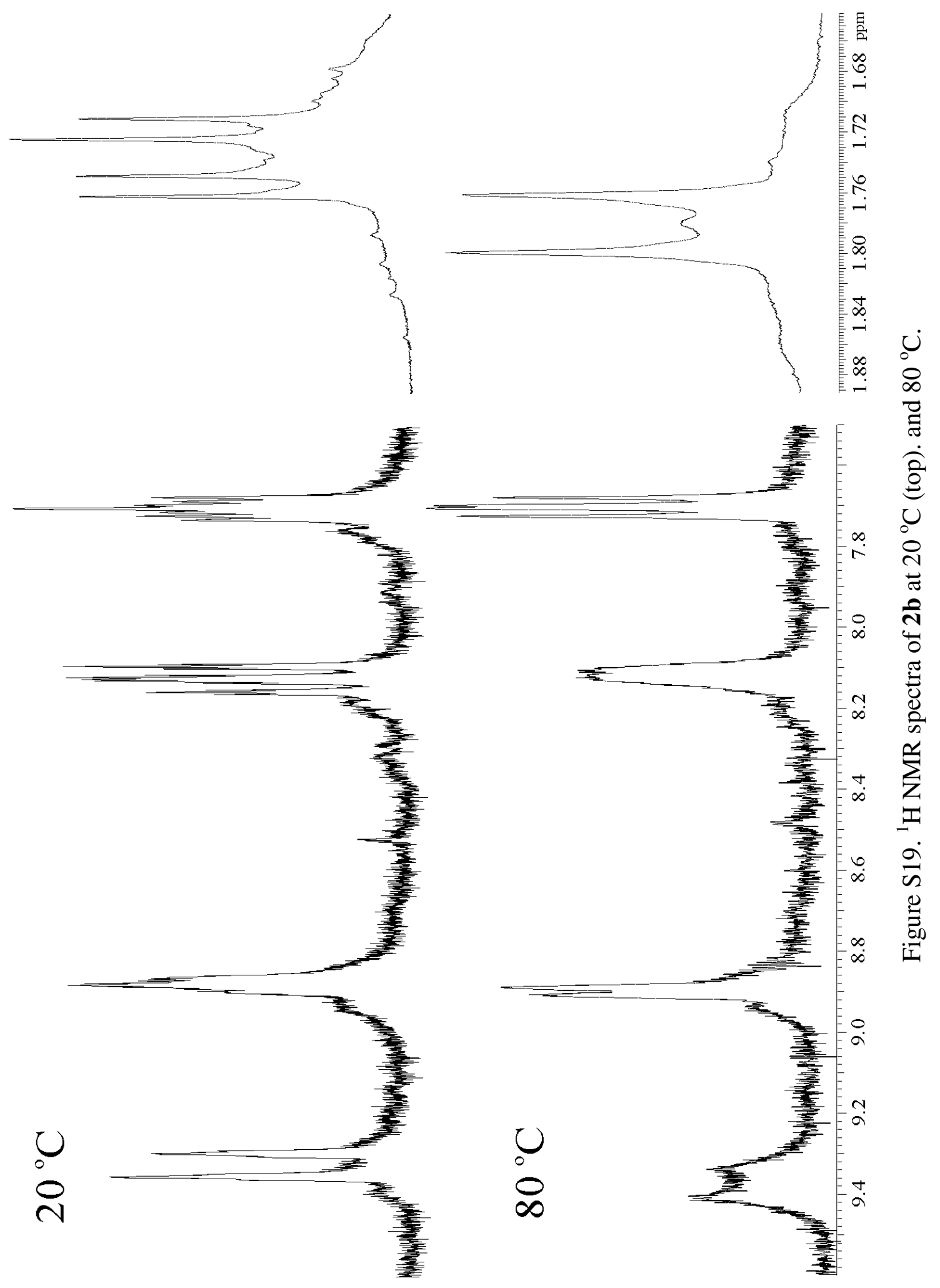


Table S1. Crystal data and structure refinement for $\mathbf{2 b}$.

Empirical formula

Formula weight

Temperature

Wavelength

Crystal system

Space group

Unit cell dimensions

Volume

$\mathrm{Z}$

Density (calculated)

Absorption coefficient

$\mathrm{F}(000)$

Crystal size

Theta range for data collection

Index ranges

Reflections collected

Independent reflections

Completeness to theta $=27.50^{\circ}$

Absorption correction

Max. and min. transmission

Refinement method

Data / restraints / parameters

Goodness-of-fit on $\mathrm{F}^{2}$

Final $\mathrm{R}$ indices [I $>2 \operatorname{sigma}(\mathrm{I})]$

$\mathrm{R}$ indices (all data)

Extinction coefficient

Largest diff. peak and hole
$\mathrm{C}_{45} \mathrm{H}_{55} \mathrm{~F}_{12} \mathrm{~N}_{5} \mathrm{O}_{14} \mathrm{P}_{4} \mathrm{Pt}_{2} \mathrm{~S}_{4}$

1760.24

150(1) K

$0.71073 \AA$

Triclinic

$P \overline{1}$

$\mathrm{a}=8.7232(3) \AA$

$\alpha=96.0857(16)^{\circ}$.

$\mathrm{b}=8.9406(2) \AA$

$\beta=99.7545(12)^{\circ}$.

$\mathrm{c}=21.7964(6) \AA$

$\gamma=100.5578(16)^{\circ}$.

1630.41(8) $\AA^{3}$

1

$1.793 \mathrm{Mg} / \mathrm{m}^{3}$

$4.606 \mathrm{~mm}^{-1}$

860

$0.30 \times 0.25 \times 0.10 \mathrm{~mm}^{3}$

2.65 to $27.50^{\circ}$.

$-10<=\mathrm{h}<=11,-11<=\mathrm{k}<=11,-28<=\mathrm{l}<=25$

10765

$7441[\mathrm{R}(\mathrm{int})=0.0231]$

$99.1 \%$

Multi-scan

0.6559 and 0.3387

Full-matrix least-squares on $\mathrm{F}^{2}$

7441 / 1 / 426

1.089

$\mathrm{R} 1=0.0382, \mathrm{wR} 2=0.0824$

$\mathrm{R} 1=0.0472, \mathrm{wR} 2=0.0870$

$0.0014(3)$

1.794 and -1.336 e. $\AA^{-3}$ 
Table S2. Atomic coordinates ( $\times 10^{4}$ ) and equivalent isotropic displacement parameters $\left(\AA^{2} \times 10^{3}\right)$ for $\mathbf{2 b}$. U(eq) is defined as one third of the trace of the orthogonalized Uij tensor.

\begin{tabular}{|c|c|c|c|c|}
\hline & $\mathrm{x}$ & $\mathrm{y}$ & $\mathrm{z}$ & $\mathrm{U}(\mathrm{eq})$ \\
\hline $\operatorname{Pt}(1)$ & 4846(1) & $434(1)$ & 2095(1) & $25(1)$ \\
\hline $\mathrm{P}(1)$ & 4994(2) & $-1705(1)$ & $1473(1)$ & $28(1)$ \\
\hline $\mathrm{P}(2)$ & $2726(2)$ & 939(1) & 1463(1) & $28(1)$ \\
\hline $\mathrm{N}(1)$ & $4875(5)$ & $2320(5)$ & $2774(2)$ & $28(1)$ \\
\hline $\mathrm{N}(2)$ & $3160(5)$ & $-206(5)$ & $7251(2)$ & $27(1)$ \\
\hline $\mathrm{C}(1)$ & $5739(7)$ & $3717(6)$ & 2779(2) & $37(1)$ \\
\hline $\mathrm{C}(2)$ & $5874(9)$ & $4875(7)$ & $3266(3)$ & $51(2)$ \\
\hline $\mathrm{C}(3)$ & $5162(9)$ & $4596(7)$ & $3767(3)$ & $53(2)$ \\
\hline $\mathrm{C}(4)$ & $4311(8)$ & $3122(6)$ & $3778(2)$ & $39(1)$ \\
\hline $\mathrm{C}(5)$ & $4178(6)$ & 2024(6) & $3266(2)$ & $31(1)$ \\
\hline$C(6)$ & $3634(8)$ & $2698(7)$ & $4306(3)$ & $43(1)$ \\
\hline $\mathrm{C}(7)$ & $3180(8)$ & $2268(7)$ & $4755(2)$ & $42(1)$ \\
\hline $\mathrm{C}(8)$ & $2724(8)$ & 1718(7) & $5275(3)$ & $40(1)$ \\
\hline $\mathrm{C}(9)$ & $2390(7)$ & $1227(7)$ & $5732(3)$ & $39(1)$ \\
\hline$C(10)$ & $2070(7)$ & $518(6)$ & $6270(2)$ & $35(1)$ \\
\hline $\mathrm{C}(11)$ & $562(7)$ & $-190(7)$ & $6330(3)$ & $43(1)$ \\
\hline $\mathrm{C}(12)$ & $381(7)$ & $-928(7)$ & 6848(3) & $43(1)$ \\
\hline $\mathrm{C}(13)$ & $1694(7)$ & $-940(6)$ & $7294(3)$ & $34(1)$ \\
\hline $\mathrm{C}(14)$ & $3348(7)$ & $498(6)$ & 6749(2) & $32(1)$ \\
\hline$C(15)$ & $6042(8)$ & $-2918(6)$ & 1919(3) & $44(1)$ \\
\hline$C(16)$ & $3161(7)$ & $-2999(6)$ & 1096(3) & $39(1)$ \\
\hline $\mathrm{C}(17)$ & $6096(7)$ & $-1316(6)$ & $857(3)$ & $35(1)$ \\
\hline $\mathrm{C}(18)$ & $862(7)$ & $-280(7)$ & $1506(3)$ & $51(2)$ \\
\hline$C(19)$ & $2442(7)$ & $2864(6)$ & $1674(3)$ & $41(1)$ \\
\hline$C(20)$ & $2759(7)$ & $873(6)$ & $635(2)$ & $37(1)$ \\
\hline$S(1)$ & $7323(2)$ & $3005(1)$ & $985(1)$ & $30(1)$ \\
\hline $\mathrm{O}(1)$ & $8420(5)$ & $2375(5)$ & $1400(2)$ & $55(1)$ \\
\hline $\mathrm{O}(2)$ & $6157(5)$ & $3589(5)$ & $1272(2)$ & $48(1)$ \\
\hline $\mathrm{O}(3)$ & $6725(5)$ & 2103(5) & $379(2)$ & $47(1)$ \\
\hline $\mathrm{F}(1)$ & $9755(5)$ & $4354(5)$ & $552(2)$ & 63(1) \\
\hline
\end{tabular}




$\begin{array}{lrlcc}\mathrm{F}(2) & 9178(6) & 5735(5) & 1307(2) & 77(1) \\ \mathrm{F}(3) & 7731(5) & 5399(4) & 386(2) & 60(1) \\ \mathrm{C}(21) & 8555(7) & 4718(7) & 803(3) & 40(1) \\ \mathrm{S}(2) & 2201(3) & 7294(2) & 3022(1) & 75(1) \\ \mathrm{O}(4) & 2856(9) & 6547(8) & 2568(3) & 105(2) \\ \mathrm{O}(5) & 3397(14) & 7370(20) & 3654(8) & 85(5) \\ \mathrm{O}(6) & 1835(19) & 8669(11) & 2939(9) & 75(4) \\ \mathrm{F}(4) & -164(7) & 6463(7) & 3557(3) & 111(2) \\ \mathrm{F}(5) & 770(40) & 4669(16) & 3194(17) & 158(14) \\ \mathrm{F}(6) & -390(30) & 6210(50) & 2614(14) & 216(18) \\ \mathrm{C}(22) & 580(11) & 5940(11) & 3105(5) & 77(2) \\ \mathrm{O}\left(5^{\prime}\right) & 2620(30) & 8290(40) & 3460(11) & 203(16) \\ \mathrm{O}\left(6^{\prime}\right) & 1000(50) & 8110(50) & 2630(18) & 240(17) \\ \mathrm{F}\left(5^{\prime}\right) & 1160(40) & 4820(30) & 3404(14) & 152(12) \\ \mathrm{F}\left(6^{\prime}\right) & -430(30) & 5330(40) & 2629(9) & 166(15) \\ \mathrm{O}^{\prime}(7) & 7810(30) & 2900(30) & 5000(13) & 196(10) \\ \mathrm{O}(8) & 7990(20) & 3050(20) & 4101(9) & 128(6) \\ \mathrm{N}(3) & 8630(30) & 3340(30) & 4648(12) & 122(7) \\ \mathrm{C}(23) & 10180(50) & 4470(60) & 4910(40) & 300(30) \\ & & & & \end{array}$


Table S3. Bond lengths $[\AA]$ and angles $\left[{ }^{\circ}\right]$ for $\mathbf{2 b}$.

\begin{tabular}{ll}
\hline $\mathrm{Pt}(1)-\mathrm{N}(2) \# 1$ & $2.110(4)$ \\
$\mathrm{Pt}(1)-\mathrm{N}(1)$ & $2.116(4)$ \\
$\mathrm{Pt}(1)-\mathrm{P}(2)$ & $2.2578(13)$ \\
$\mathrm{Pt}(1)-\mathrm{P}(1)$ & $2.2616(12)$ \\
$\mathrm{P}(1)-\mathrm{C}(15)$ & $1.801(6)$ \\
$\mathrm{P}(1)-\mathrm{C}(16)$ & $1.802(6)$ \\
$\mathrm{P}(1)-\mathrm{C}(17)$ & $1.806(5)$ \\
$\mathrm{P}(2)-\mathrm{C}(20)$ & $1.805(5)$ \\
$\mathrm{P}(2)-\mathrm{C}(19)$ & $1.806(5)$ \\
$\mathrm{P}(2)-\mathrm{C}(18)$ & $1.807(6)$ \\
$\mathrm{N}(1)-\mathrm{C}(1)$ & $1.333(6)$ \\
$\mathrm{N}(1)-\mathrm{C}(5)$ & $1.346(6)$ \\
$\mathrm{N}(2)-\mathrm{C}(14)$ & $1.338(6)$ \\
$\mathrm{N}(2)-\mathrm{C}(13)$ & $1.349(7)$ \\
$\mathrm{N}(2)-\mathrm{Pt}(1) \# 1$ & $2.110(4)$ \\
$\mathrm{C}(1)-\mathrm{C}(2)$ & $1.376(8)$ \\
$\mathrm{C}(2)-\mathrm{C}(3)$ & $1.368(9)$ \\
$\mathrm{C}(3)-\mathrm{C}(4)$ & $1.394(8)$ \\
$\mathrm{C}(4)-\mathrm{C}(5)$ & $1.379(7)$ \\
$\mathrm{C}(4)-\mathrm{C}(6)$ & $1.435(8)$ \\
$\mathrm{C}(6)-\mathrm{C}(7)$ & $1.191(8)$ \\
$\mathrm{C}(7)-\mathrm{C}(8)$ & $1.372(8)$ \\
$\mathrm{C}(8)-\mathrm{C}(9)$ & $1.189(8)$ \\
$\mathrm{C}(9)-\mathrm{C}(10)$ & $1.434(7)$ \\
$\mathrm{C}(10)-\mathrm{C}(11)$ & $1.386(8)$ \\
$\mathrm{C}(10)-\mathrm{C}(14)$ & $1.396(8)$ \\
$\mathrm{C}(11)-\mathrm{C}(12)$ & $1.382(8)$ \\
$\mathrm{C}(12)-\mathrm{C}(13)$ & $1.374(8)$ \\
$\mathrm{S}(1)-\mathrm{O}(2)$ & $1.429(4)$ \\
$\mathrm{S}(1)-\mathrm{O}(3)$ & $1.432(4)$ \\
$\mathrm{S}(1)-\mathrm{O}(1)$ & $1.437(4)$ \\
$\mathrm{S}(1)-\mathrm{C}(21)$ & $1.824(6)$ \\
$\mathrm{F}(1)-\mathrm{C}(21)$ & $1.334(7)$ \\
$\mathrm{F}(2)-\mathrm{C}(21)$ & \\
&
\end{tabular}




$\begin{array}{lc}\mathrm{F}(3)-\mathrm{C}(21) & 1.330(7) \\ \mathrm{S}(2)-\mathrm{O}\left(5^{\prime}\right) & 1.194(19) \\ \mathrm{S}(2)-\mathrm{O}(6) & 1.348(10) \\ \mathrm{S}(2)-\mathrm{O}(4) & 1.394(6) \\ \mathrm{S}(2)-\mathrm{O}(5) & 1.567(15) \\ \mathrm{S}(2)-\mathrm{O}\left(6^{\prime}\right) & 1.57(4) \\ \mathrm{S}(2)-\mathrm{C}(22) & 1.733(9) \\ \mathrm{F}(4)-\mathrm{C}(22) & 1.357(10) \\ \mathrm{F}(5)-\mathrm{C}(22) & 1.21(2) \\ \mathrm{F}(6)-\mathrm{C}(22) & 1.32(3) \\ \mathrm{C}(22)-\mathrm{F}\left(6^{\prime}\right) & 1.243(19) \\ \mathrm{C}(22)-\mathrm{F}\left(5^{\prime}\right) & 1.37(2) \\ \mathrm{O}(7)-\mathrm{N}(3) & 1.18(3) \\ \mathrm{O}(8)-\mathrm{N}(3) & 1.21(2) \\ \mathrm{N}(3)-\mathrm{C}(23) & 1.52(2) \\ & \\ \mathrm{N}(2) \# 1-\mathrm{Pt}(1)-\mathrm{N}(1) & 82.15(16) \\ \mathrm{N}(2) \# 1-\mathrm{Pt}(1)-\mathrm{P}(2) & 173.43(11) \\ \mathrm{N}(1)-\mathrm{Pt}(1)-\mathrm{P}(2) & 91.37(12) \\ \mathrm{N}(2) \# 1-\mathrm{Pt}(1)-\mathrm{P}(1) & 90.91(11) \\ \mathrm{N}(1)-\mathrm{Pt}(1)-\mathrm{P}(1) & 172.50(11) \\ \mathrm{P}(2)-\mathrm{Pt}(1)-\mathrm{P}(1) & 95.62(5) \\ \mathrm{C}(15)-\mathrm{P}(1)-\mathrm{C}(16) & 102.7(3) \\ \mathrm{C}(15)-\mathrm{P}(1)-\mathrm{C}(17) & 104.4(3) \\ \mathrm{C}(16)-\mathrm{P}(1)-\mathrm{C}(17) & 105.9(3) \\ \mathrm{C}(15)-\mathrm{P}(1)-\mathrm{Pt}(1) & 110.84(19) \\ \mathrm{C}(16)-\mathrm{P}(1)-\mathrm{Pt}(1) & 117.89(19) \\ \mathrm{C}(17)-\mathrm{P}(1)-\mathrm{Pt}(1) & 113.66(18) \\ \mathrm{C}(20)-\mathrm{P}(2)-\mathrm{C}(19) & 102.4(3) \\ \mathrm{C}(20)-\mathrm{P}(2)-\mathrm{C}(18) & 105.5(3) \\ \mathrm{C}(19)-\mathrm{P}(2)-\mathrm{C}(18) & 104.2(3) \\ \mathrm{C}(20)-\mathrm{P}(2)-\mathrm{Pt}(1) & 117.87(19) \\ \mathrm{C}(19)-\mathrm{P}(2)-\mathrm{Pt}(1) & 111.9(2) \\ \mathrm{C}(18)-\mathrm{P}(2)-\mathrm{Pt}(1) & 113.6(2) \\ \mathrm{C}(1)-\mathrm{N}(1)-\mathrm{C}(5) & 119.4(4) \\ \mathrm{C}(1)-\mathrm{N}(1)-\mathrm{Pt}(1) & 122.5(3) \\ & \\ & \end{array}$




$\begin{array}{lr}\mathrm{C}(5)-\mathrm{N}(1)-\mathrm{Pt}(1) & 117.4(3) \\ \mathrm{C}(14)-\mathrm{N}(2)-\mathrm{C}(13) & 119.2(5) \\ \mathrm{C}(14)-\mathrm{N}(2)-\mathrm{Pt}(1) \# 1 & 120.5(4) \\ \mathrm{C}(13)-\mathrm{N}(2)-\mathrm{Pt}(1) \# 1 & 119.8(3) \\ \mathrm{N}(1)-\mathrm{C}(1)-\mathrm{C}(2) & 121.0(5) \\ \mathrm{C}(3)-\mathrm{C}(2)-\mathrm{C}(1) & 120.3(6) \\ \mathrm{C}(2)-\mathrm{C}(3)-\mathrm{C}(4) & 119.0(5) \\ \mathrm{C}(5)-\mathrm{C}(4)-\mathrm{C}(3) & 117.9(5) \\ \mathrm{C}(5)-\mathrm{C}(4)-\mathrm{C}(6) & 119.4(5) \\ \mathrm{C}(3)-\mathrm{C}(4)-\mathrm{C}(6) & 122.7(5) \\ \mathrm{N}(1)-\mathrm{C}(5)-\mathrm{C}(4) & 122.4(5) \\ \mathrm{C}(7)-\mathrm{C}(6)-\mathrm{C}(4) & 173.8(7) \\ \mathrm{C}(6)-\mathrm{C}(7)-\mathrm{C}(8) & 176.5(7) \\ \mathrm{C}(9)-\mathrm{C}(8)-\mathrm{C}(7) & 177.3(7) \\ \mathrm{C}(8)-\mathrm{C}(9)-\mathrm{C}(10) & 174.3(6) \\ \mathrm{C}(11)-\mathrm{C}(10)-\mathrm{C}(14) & 118.2(5) \\ \mathrm{C}(11)-\mathrm{C}(10)-\mathrm{C}(9) & 123.4(5) \\ \mathrm{C}(14)-\mathrm{C}(10)-\mathrm{C}(9) & 118.4(5) \\ \mathrm{C}(12)-\mathrm{C}(11)-\mathrm{C}(10) & 119.2(5) \\ \mathrm{C}(13)-\mathrm{C}(12)-\mathrm{C}(11) & 119.6(5) \\ \mathrm{N}(2)-\mathrm{C}(13)-\mathrm{C}(12) & 121.6(5) \\ \mathrm{N}(2)-\mathrm{C}(14)-\mathrm{C}(10) & 122.1(5) \\ \mathrm{O}(2)-\mathrm{S}(1)-\mathrm{O}(3) & 114.9(3) \\ \mathrm{O}(2)-\mathrm{S}(1)-\mathrm{O}(1) & 114.6(3) \\ \mathrm{O}(3)-\mathrm{S}(1)-\mathrm{O}(1) & 114.7(3) \\ \mathrm{O}(2)-\mathrm{S}(1)-\mathrm{C}(21) & 103.8(3) \\ \mathrm{O}(3)-\mathrm{S}(1)-\mathrm{C}(21) & 102.8(3) \\ \mathrm{O}(1)-\mathrm{S}(1)-\mathrm{C}(21) & 103.7(3) \\ \mathrm{F}(2)-\mathrm{C}(21)-\mathrm{F}(3) & 108.0(5) \\ \mathrm{F}(2)-\mathrm{C}(21)-\mathrm{F}(1) & 107.4(5) \\ \mathrm{F}(3)-\mathrm{C}(21)-\mathrm{F}(1) & 106.9(5) \\ \mathrm{F}(2)-\mathrm{C}(21)-\mathrm{S}(1) & 112.1(4) \\ \mathrm{F}(3)-\mathrm{C}(21)-\mathrm{S}(1) & 111.6(4) \\ \mathrm{F}(1)-\mathrm{C}(21)-\mathrm{S}(1) & 110.7(4) \\ \mathrm{O}(5)-\mathrm{S}(2)-\mathrm{O}(6) & 62.9(19) \\ \mathrm{O}(5)-\mathrm{S}(2)-\mathrm{O}(4) & 138.6(11) \\ & \end{array}$




$\begin{array}{lc}\mathrm{O}(6)-\mathrm{S}(2)-\mathrm{O}(4) & 119.0(7) \\ \mathrm{O}\left(5^{\prime}\right)-\mathrm{S}(2)-\mathrm{O}(5) & 50(2) \\ \mathrm{O}(6)-\mathrm{S}(2)-\mathrm{O}\left(5^{\prime}\right) & 112.2(11) \\ \mathrm{O}(4)-\mathrm{S}(2)-\mathrm{O}\left(5^{\prime}\right) & 104.5(6) \\ \mathrm{O}\left(5^{\prime}\right)-\mathrm{S}(2)-\mathrm{O}\left(6^{\prime}\right) & 96(2) \\ \mathrm{O}(6)-\mathrm{S}(2)-\mathrm{O}\left(6^{\prime}\right) & 36.2(14) \\ \mathrm{O}(4)-\mathrm{S}(2)-\mathrm{O}\left(6^{\prime}\right) & 103.7(15) \\ \mathrm{O}(5)-\mathrm{S}(2)-\mathrm{O}\left(6^{\prime}\right) & 146.4(16) \\ \mathrm{O}\left(5^{\prime}\right)-\mathrm{S}(2)-\mathrm{C}(22) & 112.6(10) \\ \mathrm{O}(6)-\mathrm{S}(2)-\mathrm{C}(22) & 112.7(7) \\ \mathrm{O}(4)-\mathrm{S}(2)-\mathrm{C}(22) & 104.2(5) \\ \mathrm{O}(5)-\mathrm{S}(2)-\mathrm{C}(22) & 102.6(8) \\ \mathrm{O}\left(6^{\prime}\right)-\mathrm{S}(2)-\mathrm{C}(22) & 87.7(17) \\ \mathrm{F}(5)-\mathrm{C}(22)-\mathrm{F}\left(6^{\prime}\right) & 88(2) \\ \mathrm{F}(5)-\mathrm{C}(22)-\mathrm{F}(6) & 124(2) \\ \mathrm{F}\left(6^{\prime}\right)-\mathrm{C}(22)-\mathrm{F}(6) & 36(2) \\ \mathrm{F}(5)-\mathrm{C}(22)-\mathrm{F}(4) & 107.4(16) \\ \mathrm{F}\left(6^{\prime}\right)-\mathrm{C}(22)-\mathrm{F}(4) & 109.2(16) \\ \mathrm{F}(6)-\mathrm{C}(22)-\mathrm{F}(4) & 97.6(16) \\ \mathrm{F}(5)-\mathrm{C}(22)-\mathrm{F}\left(5^{\prime}\right) & 22(2) \\ \mathrm{F}\left(6^{\prime}\right)-\mathrm{C}(22)-\mathrm{F}\left(5^{\prime}\right) & 110(2) \\ \mathrm{F}(6)-\mathrm{C}(22)-\mathrm{F}\left(5^{\prime}\right) & 145(2) \\ \mathrm{F}(4)-\mathrm{C}(22)-\mathrm{F}\left(5^{\prime}\right) & 97.8(13) \\ \mathrm{F}(5)-\mathrm{C}(22)-\mathrm{S}(2) & 118.9(15) \\ \mathrm{F}\left(6^{\prime}\right)-\mathrm{C}(22)-\mathrm{S}(2) & 118.8(12) \\ \mathrm{F}(6)-\mathrm{C}(22)-\mathrm{S}(2) & 95.4(17) \\ \mathrm{F}(4)-\mathrm{C}(22)-\mathrm{S}(2) & 112.0(6) \\ \mathrm{F}\left(5^{\prime}\right)-\mathrm{C}(22)-\mathrm{S}(2) & 107.4(13) \\ \mathrm{O}(7)-\mathrm{N}(3)-\mathrm{O}(8) & 115(3) \\ \mathrm{O}(7)-\mathrm{N}(3)-\mathrm{C}(23) & 119(4) \\ \mathrm{O}(8)-\mathrm{N}(3)-\mathrm{C}(23) & 125(4) \\ & \end{array}$

Symmetry transformations used to generate equivalent atoms:

\#1 -x+1,-y,-z+1 
Table S4. Anisotropic displacement parameters $\left(\AA^{2} \times 10^{3}\right)$ for $\mathbf{2 b}$. The anisotropic displacement factor exponent takes the form: $-2 \pi^{2}\left[h^{2} a^{* 2} U^{11}+\ldots+2 h k a^{*} b^{*} U^{12}\right]$

\begin{tabular}{|c|c|c|c|c|c|c|}
\hline & $\mathrm{U}^{11}$ & $\mathrm{U}^{22}$ & $\mathrm{U}^{33}$ & $\mathrm{U}^{23}$ & U13 & $\mathrm{U}^{12}$ \\
\hline $\operatorname{Pt}(1)$ & $32(1)$ & $20(1)$ & $24(1)$ & $3(1)$ & $8(1)$ & $4(1)$ \\
\hline $\mathrm{P}(1)$ & $37(1)$ & $20(1)$ & $27(1)$ & $2(1)$ & 11(1) & $5(1)$ \\
\hline $\mathrm{P}(2)$ & $27(1)$ & $26(1)$ & $32(1)$ & $2(1)$ & $7(1)$ & $4(1)$ \\
\hline $\mathrm{N}(1)$ & $36(2)$ & $24(2)$ & $24(2)$ & $2(2)$ & $5(2)$ & $4(2)$ \\
\hline $\mathrm{N}(2)$ & $32(2)$ & $26(2)$ & $24(2)$ & $3(2)$ & $7(2)$ & $6(2)$ \\
\hline $\mathrm{C}(1)$ & $55(4)$ & $25(2)$ & $30(3)$ & $5(2)$ & $9(2)$ & $4(2)$ \\
\hline $\mathrm{C}(2)$ & $86(5)$ & $27(3)$ & $32(3)$ & $1(2)$ & $7(3)$ & $-2(3)$ \\
\hline $\mathrm{C}(3)$ & $95(5)$ & $32(3)$ & $27(3)$ & $1(2)$ & $9(3)$ & $3(3)$ \\
\hline $\mathrm{C}(4)$ & $57(4)$ & $34(3)$ & $26(3)$ & $5(2)$ & $7(2)$ & $13(3)$ \\
\hline$C(5)$ & $42(3)$ & $27(2)$ & $27(2)$ & $4(2)$ & $9(2)$ & $11(2)$ \\
\hline$C(6)$ & $63(4)$ & $36(3)$ & $28(3)$ & $-2(2)$ & $8(3)$ & $12(3)$ \\
\hline$C(7)$ & $62(4)$ & $37(3)$ & $24(3)$ & $1(2)$ & $6(3)$ & $9(3)$ \\
\hline $\mathrm{C}(8)$ & $55(4)$ & $40(3)$ & $26(3)$ & $1(2)$ & $8(2)$ & $11(3)$ \\
\hline $\mathrm{C}(9)$ & $46(3)$ & $39(3)$ & $30(3)$ & $3(2)$ & $6(2)$ & $10(3)$ \\
\hline $\mathrm{C}(10)$ & $47(3)$ & $34(3)$ & $25(3)$ & $5(2)$ & $8(2)$ & $12(2)$ \\
\hline $\mathrm{C}(11)$ & $39(3)$ & $51(4)$ & $39(3)$ & $10(3)$ & $3(3)$ & 11(3) \\
\hline $\mathrm{C}(12)$ & $27(3)$ & $56(4)$ & $49(3)$ & $17(3)$ & $12(2)$ & $6(3)$ \\
\hline $\mathrm{C}(13)$ & $39(3)$ & $33(3)$ & $36(3)$ & $9(2)$ & $13(2)$ & $13(2)$ \\
\hline $\mathrm{C}(14)$ & $40(3)$ & $26(2)$ & $29(3)$ & $2(2)$ & $8(2)$ & $8(2)$ \\
\hline$C(15)$ & $68(4)$ & $31(3)$ & $39(3)$ & $5(2)$ & $13(3)$ & $26(3)$ \\
\hline $\mathrm{C}(16)$ & $47(3)$ & $25(3)$ & $42(3)$ & $-1(2)$ & $16(3)$ & $-3(2)$ \\
\hline $\mathrm{C}(17)$ & $38(3)$ & $32(3)$ & $36(3)$ & $3(2)$ & $16(2)$ & $5(2)$ \\
\hline $\mathrm{C}(18)$ & $38(3)$ & $42(3)$ & $73(5)$ & $2(3)$ & $20(3)$ & $-1(3)$ \\
\hline$C(19)$ & $36(3)$ & $31(3)$ & $54(4)$ & $3(2)$ & $5(3)$ & $13(2)$ \\
\hline $\mathrm{C}(20)$ & $39(3)$ & $39(3)$ & $29(3)$ & $6(2)$ & $-1(2)$ & $8(2)$ \\
\hline$S(1)$ & $32(1)$ & $26(1)$ & $30(1)$ & $3(1)$ & $9(1)$ & $2(1)$ \\
\hline $\mathrm{O}(1)$ & $48(3)$ & $61(3)$ & $55(3)$ & $31(2)$ & $2(2)$ & $7(2)$ \\
\hline $\mathrm{O}(2)$ & $47(2)$ & $36(2)$ & $65(3)$ & $-1(2)$ & $30(2)$ & $3(2)$ \\
\hline $\mathrm{O}(3)$ & $64(3)$ & $35(2)$ & $37(2)$ & $-4(2)$ & $7(2)$ & $2(2)$ \\
\hline $\mathrm{F}(1)$ & $49(2)$ & $83(3)$ & $73(3)$ & $37(2)$ & $37(2)$ & $16(2)$ \\
\hline $\mathrm{F}(2)$ & $86(3)$ & $61(3)$ & $60(3)$ & $-9(2)$ & $17(2)$ & $-40(2)$ \\
\hline
\end{tabular}




\begin{tabular}{lcccccc}
$\mathrm{F}(3)$ & $73(3)$ & $41(2)$ & $72(3)$ & $27(2)$ & $15(2)$ & $15(2)$ \\
$\mathrm{C}(21)$ & $46(3)$ & $36(3)$ & $39(3)$ & $8(2)$ & $13(3)$ & $3(2)$ \\
$\mathrm{S}(2)$ & $96(2)$ & $46(1)$ & $89(2)$ & $7(1)$ & $51(1)$ & $2(1)$ \\
$\mathrm{O}(4)$ & $123(6)$ & $112(5)$ & $109(5)$ & $18(4)$ & $80(5)$ & $47(5)$ \\
$\mathrm{O}(5)$ & $31(6)$ & $124(12)$ & $80(9)$ & $-31(8)$ & $-6(6)$ & $4(6)$ \\
$\mathrm{O}(6)$ & $104(10)$ & $18(4)$ & $129(12)$ & $26(6)$ & $79(10)$ & $15(5)$ \\
$\mathrm{F}(4)$ & $90(4)$ & $112(4)$ & $162(6)$ & $48(4)$ & $85(4)$ & $28(3)$ \\
$\mathrm{F}(5)$ & $134(17)$ & $13(5)$ & $330(40)$ & $13(10)$ & $90(20)$ & $-2(7)$ \\
$\mathrm{F}(6)$ & $110(20)$ & $420(50)$ & $131(18)$ & $60(30)$ & $-14(15)$ & $120(30)$ \\
$\mathrm{C}(22)$ & $70(6)$ & $71(6)$ & $87(6)$ & $0(5)$ & $29(5)$ & $-2(4)$ \\
$\mathrm{O}\left(5^{\prime}\right)$ & $120(19)$ & $300(40)$ & $109(18)$ & $-110(20)$ & $65(15)$ & $-110(20)$ \\
$\mathrm{F}\left(5^{\prime}\right)$ & $152(19)$ & $135(19)$ & $250(20)$ & $156(19)$ & $129(18)$ & $82(15)$ \\
$\mathrm{F}\left(6^{\prime}\right)$ & $111(17)$ & $250(30)$ & $56(8)$ & $-18(12)$ & $-4(9)$ & $-121(19)$ \\
& & & & & \\
\hline
\end{tabular}


Table S5. Hydrogen coordinates ( $\left.\mathrm{x} 10^{4}\right)$ and isotropic displacement parameters $\left(\AA^{2} \mathrm{x}\right.$ $10^{3}$ ) for $\mathbf{2 b .}$

\begin{tabular}{|c|c|c|c|c|}
\hline & $\mathrm{x}$ & $\mathrm{y}$ & $\mathrm{z}$ & $\mathrm{U}(\mathrm{eq})$ \\
\hline $\mathrm{H}(1)$ & 6268 & 3916 & 2441 & 44 \\
\hline $\mathrm{H}(2)$ & 6464 & 5873 & 3254 & 61 \\
\hline $\mathrm{H}(3)$ & 5247 & 5395 & 4103 & 63 \\
\hline $\mathrm{H}(5)$ & 3573 & 1023 & 3261 & 37 \\
\hline $\mathrm{H}(11)$ & -338 & -168 & 6020 & 52 \\
\hline $\mathrm{H}(12)$ & -645 & -1424 & 6894 & 51 \\
\hline $\mathrm{H}(13)$ & 1567 & -1478 & 7641 & 41 \\
\hline $\mathrm{H}(14)$ & 4384 & 999 & 6717 & 38 \\
\hline $\mathrm{H}(15 \mathrm{~A})$ & 5525 & -3165 & 2272 & 66 \\
\hline $\mathrm{H}(15 \mathrm{~B})$ & 7145 & -2380 & 2080 & 66 \\
\hline $\mathrm{H}(15 \mathrm{C})$ & 6027 & -3869 & 1647 & 66 \\
\hline $\mathrm{H}(16 \mathrm{~A})$ & 3395 & -3944 & 896 & 59 \\
\hline $\mathrm{H}(16 \mathrm{~B})$ & 2581 & -2509 & 776 & 59 \\
\hline $\mathrm{H}(16 \mathrm{C})$ & 2510 & -3249 & 1410 & 59 \\
\hline $\mathrm{H}(17 \mathrm{~A})$ & 6208 & -2288 & 633 & 53 \\
\hline $\mathrm{H}(17 \mathrm{~B})$ & 7151 & -691 & 1041 & 53 \\
\hline $\mathrm{H}(17 \mathrm{C})$ & 5526 & -759 & 561 & 53 \\
\hline $\mathrm{H}(18 \mathrm{~A})$ & 778 & -303 & 1948 & 77 \\
\hline $\mathrm{H}(18 \mathrm{~B})$ & 817 & -1324 & 1304 & 77 \\
\hline $\mathrm{H}(18 \mathrm{C})$ & -20 & 126 & 1291 & 77 \\
\hline $\mathrm{H}(19 \mathrm{~A})$ & 3393 & 3608 & 1644 & 61 \\
\hline $\mathrm{H}(19 \mathrm{~B})$ & 2258 & 2984 & 2106 & 61 \\
\hline $\mathrm{H}(19 \mathrm{C})$ & 1522 & 3044 & 1387 & 61 \\
\hline $\mathrm{H}(20 \mathrm{~A})$ & 1783 & 1129 & 417 & 55 \\
\hline $\mathrm{H}(20 \mathrm{~B})$ & 2832 & -163 & 460 & 55 \\
\hline $\mathrm{H}(20 \mathrm{C})$ & 3681 & 1616 & 577 & 55 \\
\hline $\mathrm{H}(23 \mathrm{~A})$ & 10934 & 4376 & 4626 & 456 \\
\hline $\mathrm{H}(23 \mathrm{~B})$ & 10636 & 4259 & 5326 & 456 \\
\hline $\mathrm{H}(23 \mathrm{C})$ & 9988 & 5522 & 4944 & 456 \\
\hline
\end{tabular}


Table S6. Torsion angles $\left[^{\circ}\right]$ for $\mathbf{2 b}$.

\begin{tabular}{lc}
\hline $\mathrm{N}(2) \# 1-\mathrm{Pt}(1)-\mathrm{P}(1)-\mathrm{C}(15)$ & $22.7(3)$ \\
$\mathrm{N}(1)-\mathrm{Pt}(1)-\mathrm{P}(1)-\mathrm{C}(15)$ & $0.5(10)$ \\
$\mathrm{P}(2)-\mathrm{Pt}(1)-\mathrm{P}(1)-\mathrm{C}(15)$ & $-158.1(2)$ \\
$\mathrm{N}(2) \# 1-\mathrm{Pt}(1)-\mathrm{P}(1)-\mathrm{C}(16)$ & $140.5(2)$ \\
$\mathrm{N}(1)-\mathrm{Pt}(1)-\mathrm{P}(1)-\mathrm{C}(16)$ & $118.4(9)$ \\
$\mathrm{P}(2)-\mathrm{Pt}(1)-\mathrm{P}(1)-\mathrm{C}(16)$ & $-40.2(2)$ \\
$\mathrm{N}(2) \# 1-\mathrm{Pt}(1)-\mathrm{P}(1)-\mathrm{C}(17)$ & $-94.6(2)$ \\
$\mathrm{N}(1)-\mathrm{Pt}(1)-\mathrm{P}(1)-\mathrm{C}(17)$ & $-116.7(9)$ \\
$\mathrm{P}(2)-\mathrm{Pt}(1)-\mathrm{P}(1)-\mathrm{C}(17)$ & $84.6(2)$ \\
$\mathrm{N}(2) \# 1-\mathrm{Pt}(1)-\mathrm{P}(2)-\mathrm{C}(20)$ & $126.1(10)$ \\
$\mathrm{N}(1)-\mathrm{Pt}(1)-\mathrm{P}(2)-\mathrm{C}(20)$ & $135.4(2)$ \\
$\mathrm{P}(1)-\mathrm{Pt}(1)-\mathrm{P}(2)-\mathrm{C}(20)$ & $-47.3(2)$ \\
$\mathrm{N}(2) \# 1-\mathrm{Pt}(1)-\mathrm{P}(2)-\mathrm{C}(19)$ & $7.8(10)$ \\
$\mathrm{N}(1)-\mathrm{Pt}(1)-\mathrm{P}(2)-\mathrm{C}(19)$ & $17.2(2)$ \\
$\mathrm{P}(1)-\mathrm{Pt}(1)-\mathrm{P}(2)-\mathrm{C}(19)$ & $-165.6(2)$ \\
$\mathrm{N}(2) \# 1-\mathrm{Pt}(1)-\mathrm{P}(2)-\mathrm{C}(18)$ & $-109.8(10)$ \\
$\mathrm{N}(1)-\mathrm{Pt}(1)-\mathrm{P}(2)-\mathrm{C}(18)$ & $-100.4(3)$ \\
$\mathrm{P}(1)-\mathrm{Pt}(1)-\mathrm{P}(2)-\mathrm{C}(18)$ & $76.9(2)$ \\
$\mathrm{N}(2) \# 1-\mathrm{Pt}(1)-\mathrm{N}(1)-\mathrm{C}(1)$ & $87.1(4)$ \\
$\mathrm{P}(2)-\mathrm{Pt}(1)-\mathrm{N}(1)-\mathrm{C}(1)$ & $-91.8(4)$ \\
$\mathrm{P}(1)-\mathrm{Pt}(1)-\mathrm{N}(1)-\mathrm{C}(1)$ & $109.4(9)$ \\
$\mathrm{N}(2) \# 1-\mathrm{Pt}(1)-\mathrm{N}(1)-\mathrm{C}(5)$ & $-83.1(4)$ \\
$\mathrm{P}(2)-\mathrm{Pt}(1)-\mathrm{N}(1)-\mathrm{C}(5)$ & $98.0(4)$ \\
$\mathrm{P}(1)-\mathrm{Pt}(1)-\mathrm{N}(1)-\mathrm{C}(5)$ & $-60.7(11)$ \\
$\mathrm{C}(5)-\mathrm{N}(1)-\mathrm{C}(1)-\mathrm{C}(2)$ & $-2.6(8)$ \\
$\mathrm{Pt}(1)-\mathrm{N}(1)-\mathrm{C}(1)-\mathrm{C}(2)$ & $-172.6(5)$ \\
$\mathrm{N}(1)-\mathrm{C}(1)-\mathrm{C}(2)-\mathrm{C}(3)$ & $2.2(10)$ \\
$\mathrm{C}(1)-\mathrm{C}(2)-\mathrm{C}(3)-\mathrm{C}(4)$ & $0.4(11)$ \\
$\mathrm{C}(2)-\mathrm{C}(3)-\mathrm{C}(4)-\mathrm{C}(5)$ & $-2.4(10)$ \\
$\mathrm{C}(2)-\mathrm{C}(3)-\mathrm{C}(4)-\mathrm{C}(6)$ & $175.0(6)$ \\
$\mathrm{C}(1)-\mathrm{N}(1)-\mathrm{C}(5)-\mathrm{C}(4)$ & $0.5(8)$ \\
$\mathrm{Pt}(1)-\mathrm{N}(1)-\mathrm{C}(5)-\mathrm{C}(4)$ & $171.0(4)$ \\
$\mathrm{C}(3)-\mathrm{C}(4)-\mathrm{C}(5)-\mathrm{N}(1)$ & \\
$\mathrm{C}(6)-\mathrm{C}(4)-\mathrm{C}(5)-\mathrm{N}(1)$ & \\
& \\
&
\end{tabular}




$\begin{array}{lc}\mathrm{C}(5)-\mathrm{C}(4)-\mathrm{C}(6)-\mathrm{C}(7) & 69(6) \\ \mathrm{C}(3)-\mathrm{C}(4)-\mathrm{C}(6)-\mathrm{C}(7) & -108(6) \\ \mathrm{C}(4)-\mathrm{C}(6)-\mathrm{C}(7)-\mathrm{C}(8) & -5(16) \\ \mathrm{C}(6)-\mathrm{C}(7)-\mathrm{C}(8)-\mathrm{C}(9) & 23(22) \\ \mathrm{C}(7)-\mathrm{C}(8)-\mathrm{C}(9)-\mathrm{C}(10) & -40(18) \\ \mathrm{C}(8)-\mathrm{C}(9)-\mathrm{C}(10)-\mathrm{C}(11) & -113(7) \\ \mathrm{C}(8)-\mathrm{C}(9)-\mathrm{C}(10)-\mathrm{C}(14) & 65(7) \\ \mathrm{C}(14)-\mathrm{C}(10)-\mathrm{C}(11)-\mathrm{C}(12) & -2.0(9) \\ \mathrm{C}(9)-\mathrm{C}(10)-\mathrm{C}(11)-\mathrm{C}(12) & 175.9(6) \\ \mathrm{C}(10)-\mathrm{C}(11)-\mathrm{C}(12)-\mathrm{C}(13) & 0.4(9) \\ \mathrm{C}(14)-\mathrm{N}(2)-\mathrm{C}(13)-\mathrm{C}(12) & -2.7(8) \\ \mathrm{Pt}(1) \# 1-\mathrm{N}(2)-\mathrm{C}(13)-\mathrm{C}(12) & -174.4(4) \\ \mathrm{C}(11)-\mathrm{C}(12)-\mathrm{C}(13)-\mathrm{N}(2) & 2.1(9) \\ \mathrm{C}(13)-\mathrm{N}(2)-\mathrm{C}(14)-\mathrm{C}(10) & 1.0(7) \\ \mathrm{Pt}(1) \# 1-\mathrm{N}(2)-\mathrm{C}(14)-\mathrm{C}(10) & 172.6(4) \\ \mathrm{C}(11)-\mathrm{C}(10)-\mathrm{C}(14)-\mathrm{N}(2) & 1.4(8) \\ \mathrm{C}(9)-\mathrm{C}(10)-\mathrm{C}(14)-\mathrm{N}(2) & -176.7(5) \\ \mathrm{O}(2)-\mathrm{S}(1)-\mathrm{C}(21)-\mathrm{F}(2) & 57.6(5) \\ \mathrm{O}(3)-\mathrm{S}(1)-\mathrm{C}(21)-\mathrm{F}(2) & 177.6(5) \\ \mathrm{O}(1)-\mathrm{S}(1)-\mathrm{C}(21)-\mathrm{F}(2) & -62.5(5) \\ \mathrm{O}(2)-\mathrm{S}(1)-\mathrm{C}(21)-\mathrm{F}(3) & -63.7(5) \\ \mathrm{O}(3)-\mathrm{S}(1)-\mathrm{C}(21)-\mathrm{F}(3) & 56.4(5) \\ \mathrm{O}(1)-\mathrm{S}(1)-\mathrm{C}(21)-\mathrm{F}(3) & 176.2(4) \\ \mathrm{O}(2)-\mathrm{S}(1)-\mathrm{C}(21)-\mathrm{F}(1) & 177.3(4) \\ \mathrm{O}(3)-\mathrm{S}(1)-\mathrm{C}(21)-\mathrm{F}(1) & -62.6(5) \\ \mathrm{O}(1)-\mathrm{S}(1)-\mathrm{C}(21)-\mathrm{F}(1) & 57.3(5) \\ \mathrm{O}\left(5^{\prime}\right)-\mathrm{S}(2)-\mathrm{C}(22)-\mathrm{F}(5) & -110(3) \\ \mathrm{O}(6)-\mathrm{S}(2)-\mathrm{C}(22)-\mathrm{F}(5) & -179(2) \\ \mathrm{O}(4)-\mathrm{S}(2)-\mathrm{C}(22)-\mathrm{F}(5) & 51(2) \\ \mathrm{O}(5)-\mathrm{S}(2)-\mathrm{C}(22)-\mathrm{F}(5) & -58(2) \\ \mathrm{O}\left(6^{\prime}\right)-\mathrm{S}(2)-\mathrm{C}(22)-\mathrm{F}(5) & 155(2) \\ \mathrm{O}\left(5^{\prime}\right)-\mathrm{S}(2)-\mathrm{C}(22)-\mathrm{F}\left(6^{\prime}\right) & 145(3) \\ \mathrm{O}(6)-\mathrm{S}(2)-\mathrm{C}(22)-\mathrm{F}\left(6^{\prime}\right) & 76(2) \\ \mathrm{O}(4)-\mathrm{S}(2)-\mathrm{C}(22)-\mathrm{F}\left(6^{\prime}\right) & -54(2) \\ \mathrm{O}(5)-\mathrm{S}(2)-\mathrm{C}(22)-\mathrm{F}\left(6^{\prime}\right) & \\ \mathrm{O}\left(6^{\prime}\right)-\mathrm{S}(2)-\mathrm{C}(22)-\mathrm{F}\left(6^{\prime}\right) & \\ & \end{array}$




$\begin{array}{lc}\mathrm{O}\left(5^{\prime}\right)-\mathrm{S}(2)-\mathrm{C}(22)-\mathrm{F}(6) & 117(3) \\ \mathrm{O}(6)-\mathrm{S}(2)-\mathrm{C}(22)-\mathrm{F}(6) & 48.1(19) \\ \mathrm{O}(4)-\mathrm{S}(2)-\mathrm{C}(22)-\mathrm{F}(6) & -82.3(18) \\ \mathrm{O}(5)-\mathrm{S}(2)-\mathrm{C}(22)-\mathrm{F}(6) & 168.9(18) \\ \mathrm{O}\left(6^{\prime}\right)-\mathrm{S}(2)-\mathrm{C}(22)-\mathrm{F}(6) & 21(2) \\ \mathrm{O}\left(5^{\prime}\right)-\mathrm{S}(2)-\mathrm{C}(22)-\mathrm{F}(4) & 17(2) \\ \mathrm{O}(6)-\mathrm{S}(2)-\mathrm{C}(22)-\mathrm{F}(4) & -52.4(12) \\ \mathrm{O}(4)-\mathrm{S}(2)-\mathrm{C}(22)-\mathrm{F}(4) & 177.2(7) \\ \mathrm{O}(5)-\mathrm{S}(2)-\mathrm{C}(22)-\mathrm{F}(4) & 68.4(9) \\ \mathrm{O}\left(6^{\prime}\right)-\mathrm{S}(2)-\mathrm{C}(22)-\mathrm{F}(4) & -79.2(16) \\ \mathrm{O}\left(5^{\prime}\right)-\mathrm{S}(2)-\mathrm{C}(22)-\mathrm{F}\left(5^{\prime}\right) & -90(3) \\ \mathrm{O}(6)-\mathrm{S}(2)-\mathrm{C}(22)-\mathrm{F}\left(5^{\prime}\right) & -158.7(16) \\ \mathrm{O}(4)-\mathrm{S}(2)-\mathrm{C}(22)-\mathrm{F}\left(5^{\prime}\right) & 70.9(15) \\ \mathrm{O}(5)-\mathrm{S}(2)-\mathrm{C}(22)-\mathrm{F}\left(5^{\prime}\right) & -37.9(15) \\ \mathrm{O}\left(6^{\prime}\right)-\mathrm{S}(2)-\mathrm{C}(22)-\mathrm{F}\left(5^{\prime}\right) & 174(2)\end{array}$

Symmetry transformations used to generate equivalent atoms:

\#1 -x+1,-y,-z+1 